\title{
TEMPLATE ULTRAVIOLET TO NEAR-INFRARED SPECTRA OF STAR-FORMING GALAXIES AND THEIR APPLICATION TO $K$-CORRECTIONS
}

\author{
Anne L. Kinney, ${ }^{1,3,4,5}$ Daniela Calzetti, ${ }^{1,3,4}$ Ralph C. Bohlin, ${ }^{1,4}$ Kerry McQuade, ${ }^{1}$ \\ Thaisa Storchi-BergmanN, ${ }^{2,5}$ AND Henrique R. SChmitt ${ }^{1,2,6}$ \\ Received 1994 September 23; accepted 1996 February 29
}

\begin{abstract}
Template UV-optical spectra of quiescent and starburst galaxies are presented and used to derive $K$ corrections as a function of morphological type and redshift. IUE observations and archival data are used for the UV template spectra. The optical spectra are from ground-based observations obtained in apertures that match closely the $200 \operatorname{arcsec}^{2} I U E$ aperture. The templates of quiescent galaxies are built according to morphological type, elliptical, bulge, S0, Sa, Sb, and Sc, and the templates of starburst galaxies according to color excess. The unprecedented characteristics of these templates is that UV and optical spectra have been obtained in matched apertures to produce consistent spectral information from 1200 to $10,000 \AA$. Despite the relatively small IUE aperture, the galaxy stellar populations are well represented in the elliptical, S0, Sa, and Sc, and in the starburst templates. The spectra are available digitally.

The UV-optical templates can be applied to the classification of high-redshift galaxies and to the identification of the host galaxies of quasars.

The templates predict that observed magnitudes from traditional ground-based photometric surveys can be uniquely interpreted. For example, $U, B$, and $I$ magnitudes uniquely determine both the redshift and the morphological type of a galaxy. The template spectra are also used to calculate $K$-corrections for galaxies as a function of morphological type and redshift, up to $z=2$. These improved $K$-corrections are not sufficient to explain the excess counts in faint blue galaxies.

A subset of our galaxy templates are linked with published data from the radio to the X-ray for galaxies and quasars. A comparison between the quiescent galaxies and the quasars suggests that, in the optical band, the host galaxy is a factor of 10-100 fainter in flux than the quasar.
\end{abstract}

Subject headings: galaxies: distances and redshifts - galaxies: photometry - galaxies: starburst galaxies: stellar content - quasars: general

\section{INTRODUCTION}

As searches for faint, primeval galaxies continue, the number of unresolved questions grows. Faint galaxy counts reveal more blue galaxies than predicted based on local galaxy counts; however, the predicted number of red galaxies is about right. The number counts raise the question of the relation between local galaxies and early galaxies: did the numerous early galaxies merge to create present-day galaxies as suggested by Broadhurst, Ellis, \& Glazebrook (1992), or did an initial burst of star formation make small galaxies temporarily bright? When did the first galaxies form, and what is the best method to find these primeval galaxies? More specifically, how does the first epoch of star formation compare with later epochs, where the stars are made of processed gas?

Here, we produce spectral energy distributions (SEDs) for local galaxies from the ultraviolet to the near-infrared, in order to relate morphological type to spectral energy dis-

\footnotetext{
${ }^{1}$ Space Telescope Science Institute, 3700 San Martin Drive, Baltimore, MD 21218.

${ }^{2}$ Instituto de Física, Universidade Federal Rio Grande do Sul C.P. 15051, Porto Alegre, RS, 91501-970, Brazil.

${ }^{3}$ Visiting Astronomers, Kitt Peak National Observatory, National Optical Astronomy Observatories, which is operated by AURA, Inc., under a cooperative agreement with the National Science Foundation.

${ }^{4}$ Guest Investigators with the IUE Observatory.

5 Visiting Astronomers, Cerro Tololo Inter-American Observatory (CTIO), National Optical Astronomy Observatories, which is operated by AURA, Inc., under a cooperative agreement with the National Science Foundation.

${ }^{6} \mathrm{CNPq}$ fellow.
}

tribution. Observations in the ultraviolet allow corrections to colors ( $K$-corrections) to be calculated as a function of redshift up to $z \sim 2$ for each morphological class.

A large aperture spectrum of a galaxy contains elements from physically different regions of the galaxy and is more complex than the spectrum of a single star. Dust extinction complicates the interpretation of the spectrum, since a dusty galaxy is expected to be relatively opaque in the UV and relatively transparent in the IR.

The contributors to a galaxy's UV, optical, and near-IR spectrum are hot stars, cool stars, $\mathrm{H}$ iI regions, and the interstellar medium (ISM). The wavelength region shortward of $912 \AA$ is dominated by O star emission and interstellar absorption by $\mathrm{H}$ I. Early-type $\mathrm{OB}$ stars are responsible for the UV continuum and absorption lines longward of $1200 \AA$. Cool stars contribute the longer wavelength continuum and most of the long wavelength absorption lines. The UV to IR spectrum samples a wide range in population from young (less than $10^{7} \mathrm{yr}$ ) to old stars. H II regions are evidenced by the presence of emission lines, and the ISM creates certain absorption lines (e.g., Tables 4-7 in Kinney et al. 1993).

We present template spectra of elliptical, bulge, S0, Sa, $\mathrm{Sb}, \mathrm{Sc}$, and starburst galaxies in a matched $10^{\prime \prime} \times 20^{\prime \prime}$ aperture from $1200 \AA$ to $8000 \AA$, and in some cases to $10,000 \AA$. The observations are presented in $\S 2$, and the procedure adopted to produce the templates in $\S 3$. The general properties of the templates are discussed in $\S 4.1$. Applications of the templates to the classification of high-redshift galaxies and to the identification of the host galaxies of 
TABLE 1

Atlas GalaXies

\begin{tabular}{|c|c|c|c|c|c|c|c|c|c|}
\hline Site & $I U E$ & $\begin{array}{c}\text { Galaxy } \\
\text { Name }\end{array}$ & $\begin{array}{c}\text { Morphological } \\
\text { Type }\end{array}$ & Radius & $\begin{array}{c}v_{H} \\
\left(\mathrm{~km} \mathrm{~s}^{-1}\right)\end{array}$ & $B_{T}$ & $\begin{array}{c}M_{B} \\
\left(H_{0}=50\right)\end{array}$ & $E(B-V)_{\mathrm{MW}}$ & $\begin{array}{l}\text { Other } \\
\text { Names }\end{array}$ \\
\hline CTIO & $S+L$ & NGC 210 & $\mathrm{Sb}$ & $2^{\prime} 30^{\prime \prime}$ & 1768 & 11.65 & -21.86 & 0.01 & \\
\hline KPNO & $S+L$ & NGC 224 & $\mathrm{Sb}$ & 8000 & -297 & 4.38 & -21.61 & 0.08 & M31 \\
\hline KPNO & $S+\bar{L}$ & NGC 598 & Scd & 3524 & -180 & 6.26 & -19.07 & 0.04 & M33 \\
\hline KPNO & $\mathrm{L}$ & NGC 1023 & SB0 & 433 & 661 & 10.36 & -21.17 & 0.06 & \\
\hline & $S+L$ & NGC 1058 & Sc & 130 & 518 & 12.15 & -19.27 & 0.06 & \\
\hline CTIO & $S+L$ & NGC 1399 & E1 pec & 327 & 1465 & 10.79 & -21.61 & 0.00 & \\
\hline CTIO & $S+L$ & NGC 1404 & $\mathrm{E} 2$ & 139 & 1994 & 11.06 & -21.34 & 0.00 & \\
\hline CTIO & $S+L$ & NGC 1433 & SBab & 315 & 1061 & 10.68 & -21.15 & 0.00 & \\
\hline CTIO & $S+L$ & NGC 1553 & S0 pec & 215 & 1236 & 10.42 & -21.26 & 0.00 & \\
\hline & $S+L$ & NGC 1637 & $\mathrm{Sc}$ & 200 & 717 & 11.82 & -19.72 & 0.03 & \\
\hline KPNO & $\mathrm{S}+\mathrm{L}$ & NGC 2403 & Sc & 1057 & 131 & 8.89 & -19.47 & 0.04 & \\
\hline$\ldots$ & $S+L$ & NGC 2681 & $\mathrm{Sa}$ & 148 & 715 & 11.09 & -20.48 & 0.02 & \\
\hline$\ldots$ & $S+\bar{L}$ & NGC 2841 & $\mathrm{Sb}$ & 403 & 637 & 10.17 & -21.53 & 0.00 & \\
\hline KPNO & $S+\bar{L}$ & NGC 3031 & $\mathrm{Sb}$ & 1327 & -36 & 7.86 & -20.75 & 0.04 & M81 \\
\hline$\ldots$ & $\mathrm{S}$ & NGC 3432 & $\mathrm{Sc}$ & 324 & 616 & 11.73 & -19.50 & 0.00 & \\
\hline$\ldots$ & $S+L$ & NGC 3994 & $\mathrm{Sc}$ & 30 & 3096 & 13.30 & -20.81 & 0.01 & \\
\hline$\ldots$ & $\mathrm{L}$ & NGC 4102 & $\mathrm{Sb}$ & 130 & 865 & 12.30 & -19.80 & 0.00 & \\
\hline $\begin{array}{l}\cdots \\
\ldots\end{array}$ & $S+L$ & NGC 4259 & $\mathrm{Sc}$ & 36 & 2487 & 14.55 & & 0.00 & \\
\hline$\ldots$ & $S+L$ & NGC 4314 & SBa pec & 206 & 883 & 11.35 & -19.80 & 0.02 & \\
\hline$\ldots$ & $\mathrm{L}$ & NGC 4350 & S0 & 130 & 1184 & 11.88 & -19.82 & 0.01 & \\
\hline$\cdots$ & $S+L$ & NGC 4382 & S0 pec & 333 & 739 & 10.10 & -21.60 & 0.01 & M85 \\
\hline CTIO & $\mathrm{L}$ & NGC 4569 & SABab & 445 & -261 & 10.23 & -22.31 & 0.02 & M90 \\
\hline CTIO & $S+L$ & NGC 4594 & $\mathrm{Sa}$ & 421 & 1089 & 9.28 & -22.81 & 0.03 & M104 \\
\hline$\ldots$ & $S+L$ & NGC 4736 & SAab & 536 & 311 & 8.92 & -20.81 & 0.00 & M94 \\
\hline$\ldots$ & $S+L$ & NGC 4826 & $\mathrm{Sb}$ & 500 & 413 & 9.37 & -20.61 & 0.04 & M64 \\
\hline$\ldots$ & $S+L$ & NGC 5194 & Sbc & 536 & 464 & 8.98 & -21.60 & 0.00 & M51a \\
\hline KPNO & $S+L$ & NGC 6340 & $\mathrm{SA}(\mathrm{s}) 0 / \mathrm{a}$ & 142 & 1234 & 11.90 & -21.05 & 0.05 & \\
\hline CTIO & $\mathrm{S}$ & NGC 6868 & E2 & 145 & 2764 & 11.83 & -21.93 & 0.04 & \\
\hline CTIO & $S+L$ & NGC 7083 & $\mathrm{Sb}$ & 157 & 3089 & 11.80 & -22.81 & 0.02 & \\
\hline CTIO & $S+L$ & NGC 7196 & E3 & 115 & 2981 & 12.46 & -21.37 & 0.00 & \\
\hline
\end{tabular}

Notes.-The first column lists the observatory where the optical spectra for each galaxy were obtained (KPNO or CTIO). The second column specifies if the UV spectra consisted of data from both the IUE Short-Wavelength and Long-Wavelength Cameras $(\mathrm{S}+\mathrm{L})$ or from one of the two (S or $\mathrm{L})$. The morphological type follows, where possible, the classical Hubble classification. The radius is half the largest diameter for the galaxy as given in NED. The heliocentric velocity $\left(v_{H}\right)$ and the apparent magnitude $\left(B_{T}\right)$ are from the Revised Shapley-Ames Catalogue (RSA), for from The Third Reference Catalogue of Bright Galaxies (RC3). $M_{B}$ is the absolute magnitude calculated from the apparent magnitude corrected for Galactic absorption and from the recession velocity reduced to the centroid of the Local Group (cf. RSA). The reddening $E(B-V)_{\mathrm{MW}}$ from our Galaxy is from Burstein \& Heiles 1984.

quasars are shown in $\S \S 4.2$ and 4.3 , respectively. Colorcolor diagrams and $K$-corrections are produced in $\S 4.4$. Finally, we compare our observed SEDs with those produced by stellar population models in $\S 4.5$.

The template spectral energy distributions and their $1 \sigma$ standard deviations are presented in the Appendix, along with averages and standard deviations in $200 \AA$ bins. Diagrams of color versus redshift and color versus color for the morphological types are also presented in the Appendix.

\section{OBSERVATIONS AND DATA ANALYSIS}

The template SEDs are derived from the UV and optical spectra of the central regions of 30 quiescent galaxies, which cover morphological types from elliptical to Sc, and of the central regions of 39 starburst galaxies, which are mainly irregular and galaxies with disturbed morphologies (Calzetti, Kinney, \& Storchi-Bergmann 1994; McQuade, Calzetti, \& Kinney 1995; Storchi-Bergmann, Kinney, \& Challis 1995).

The UV spectra for six of the quiescent galaxies were taken with the $I U E$ during observing campaigns in 1992 and 1993 as part of a program to produce templates of normal galaxies. The remaining UV spectra are archival $I U E$ spectra collected in the atlas of Kinney et al. (1993) and the atlas of Koratkar et al. (1996). The UV spectra cover the wavelength range 1200-3200 $\AA$ with a resolution of about $6 \AA$. The UV spectra used in the templates are corrected for Galactic extinction based on the $E(B-V)$ values listed in Table 1 of Kinney et al. (1993) and using the UV extinction curve of Seaton (1979). In all but one spectrum, NGC 1569, this effect is very small. Approximately half the optical spectra were obtained at the CTIO $1 \mathrm{~m}$ telescope with the two-dimensional Frutti detector for the visible range and at the $1.5 \mathrm{~m}$ telescope with a CCD detector for the near-IR wavelength range (Storchi-Bergmann et al. 1995). A long slit with a $10^{\prime \prime}$ width was used, and a window $20^{\prime \prime}$ long was extracted to match the $200 \operatorname{arcsec}^{2}$ IUE aperture. The CTIO spectra cover the wavelength range $3200-10,000 \AA$ with a resolution $\sim 8 \AA$. The remaining optical spectra were obtained at the KPNO $0.9 \mathrm{~m}$ with the intensified Reticon spectrograph, using a circular aperture of 13.5 diameter. The KPNO spectra cover the wavelength range 3200-7700 $\AA$ with a resolution of $10 \AA$ (McQuade et al. 1995). The difference in flux attributable to the difference in size between the optical and the IUE apertures is the difference in size convolved with the flux distribution within the aperture and is therefore less than $30 \%$, which is within the uncertainty of the absolute calibration of the optical fluxes, so that no normalization has been done. Details on the reduction and calibration of the spectra can be found in 
Kinney et al. (1993), McQuade et al. (1995), and StorchiBergmann et al. (1995).

Table 1 lists the 30 quiescent galaxies used in the elliptical and spiral templates, along with the galaxy name, the morphological type, the size, the redshift, the apparent and absolute blue magnitudes $\left(H_{0}=50 \mathrm{~km} \mathrm{~s}^{-1} \mathrm{Mpc}^{-1}\right)$, and the foreground reddening from the Milky Way $\left[E(B-V)_{\mathrm{MW}}\right.$; see Burstein \& Heiles 1984]. Comparable information on the 39 starburst galaxies can be found in Calzetti et al. (1994).

\section{THE TEMPLATES}

\subsection{How the Templates Are Built}

The quiescent galaxies are grouped according to morphological type: E (elliptical), S0, Sa, Sb, and Sc, respectively, as shown in Table 2. The UV and optical spectra of the Sb galaxies NGC 224 (M31) and NGC 3031 (M81) are dominated by the stellar population in the bulge, and therefore the two galaxies form a separate group, the bulge template. The starburst galaxies are grouped in six classes

TABLE 2

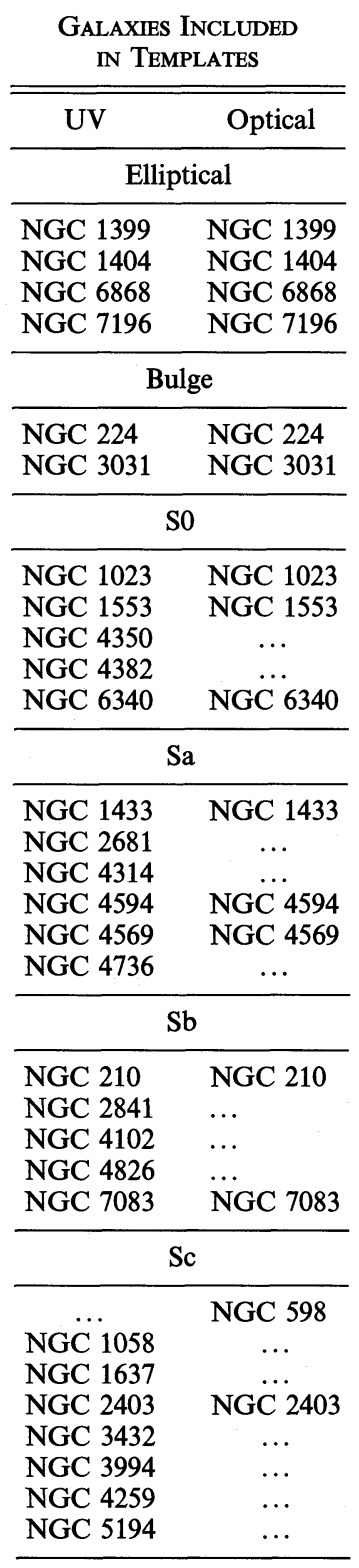

according to increasing values of the intrinsic color excess $E(B-V)$, as derived from the ratio of the Balmer emission lines $\mathrm{H} \alpha / \mathrm{H} \beta$ : from $E(B-V) \simeq 0.05$ to $E(B-V) \simeq 0.65$ (see Table 3 in Calzetti et al. 1994). Calzetti et al. (1994) established that dust obscuration, i.e., the intrinsic color excess, is the dominant parameter that determines the appearance of the starburst SED.

The template for the elliptical galaxies includes only those galaxies that have a narrow range of $\mathrm{Mg} 2$ indexes and comparable absolute magnitudes to avoid the well-known color-luminosity relation of ellipticals. The $\mathrm{Mg} 2$ indexes of the included galaxies are NGC $1399=0.334$, NGC $1404=0.317$, NGC $6868=0.317$, and NGC $7196=0.286$ (Burstein et al. 1988). In Table A1, it can be seen that the elliptical template has a low signal-to noise ratio $(\mathrm{S} / \mathrm{N})$. The $1 \sigma$ standard deviation quoted in Table A1 is dominated by the uncertainty in the spectra rather than in the intrinsic differences between the galaxies. To demonstrate this, note that the average fluxes, standard deviations, and signal-tonoise ratios for the individual galaxies in the spectral regions $1500-1900$ are NGC $1399, F_{\lambda}=0.070 \pm 0.014$, $\mathrm{S} / \mathrm{N}=5.1 ; \quad \mathrm{NGC} 1404, \quad F_{\lambda}=0.035 \pm 0.039, \quad \mathrm{~S} / \mathrm{N}=0.9 ;$ NGC 6868, $F_{\lambda}=0.052 \pm 0.076, \mathrm{~S} / \mathrm{N}=0.7$; and NGC 7196, $F_{\lambda}=0.112 \pm 0.109, \mathrm{~S} / \mathrm{N}=1.0$. The details of the individual spectra will be presented in Koratkar et al. (1996).

To what degree are the templates affected by aperture effects? The differences in SEDs of individual galaxies do not show evidence of being correlated to the size or distance of the galaxy, as would be expected if the galaxies' spectra were dominated by aperture effects. For example, the galaxies making up the Sa template, NGC 1433, NGC 2681, NGC 4314, NGC 4594, and NGC 4736, have a factor of over 2 in galaxy size, with the galaxies having radii of $3^{\prime} 15^{\prime \prime}$, $1^{\prime} 48^{\prime \prime}, 2^{\prime} 06^{\prime \prime}, 4^{\prime} 21^{\prime \prime}$, and $5^{\prime} 36^{\prime \prime}$, respectively, but only a small range of distance to the galaxy $(18.5,16,17,17.5$, and 6.9 Mpc, respectively). Meanwhile, their UV slopes (with values $-0.95 \pm 0.28,0.17 \pm 0.40,-0.07 \pm 0.35,-1.23 \pm 0.44$, and $0.27 \pm 0.17$, respectively), which serve as a rough measure of their overall SED, do not track with galaxy radius or show a trend for distant galaxies to be bluer than nearby galaxies, or a trend for small galaxies to be bluer than large galaxies, as would be the case if the individual SEDs were dominated by aperture effects. For example, NGC 1433 and NGC 4314 have similar radii $\left(3^{\prime} 15^{\prime \prime}\right.$ and $2^{\prime} 06^{\prime \prime}$, respectively) and similar distances (18.5 and 17.0 Mpc, respectively), but have very different UV slopes $(-0.95 \pm 0.28$ and $0.07 \pm 0.35)$. The differences between galaxies thus appear to be due to intrinsic differences rather than to aperture effects.

In order to make the templates, all the spectra are shifted to the rest frame and corrected for the foreground Galactic extinction using the Seaton (1979) extinction curve. Within each starburst group, the UV-optical spectra are rescaled to a common flux value and are averaged, after weighting each spectrum by its signal-to-noise ratio, to produce the final template. The templates of the quiescent galaxies are constructed by averaging separately the UV and optical spectra, since not all the quiescent galaxies observed at UV wavelengths were observed in the optical as well (Table 2). Within each morphological group, the optical spectra are averaged after rescaling the fluxes to a common value and weighting each spectrum by the exposure time. This is analogous to the $\mathrm{S} / \mathrm{N}$ weighting procedure, since the optical spectra of the quiescent galaxies do not show a large range 


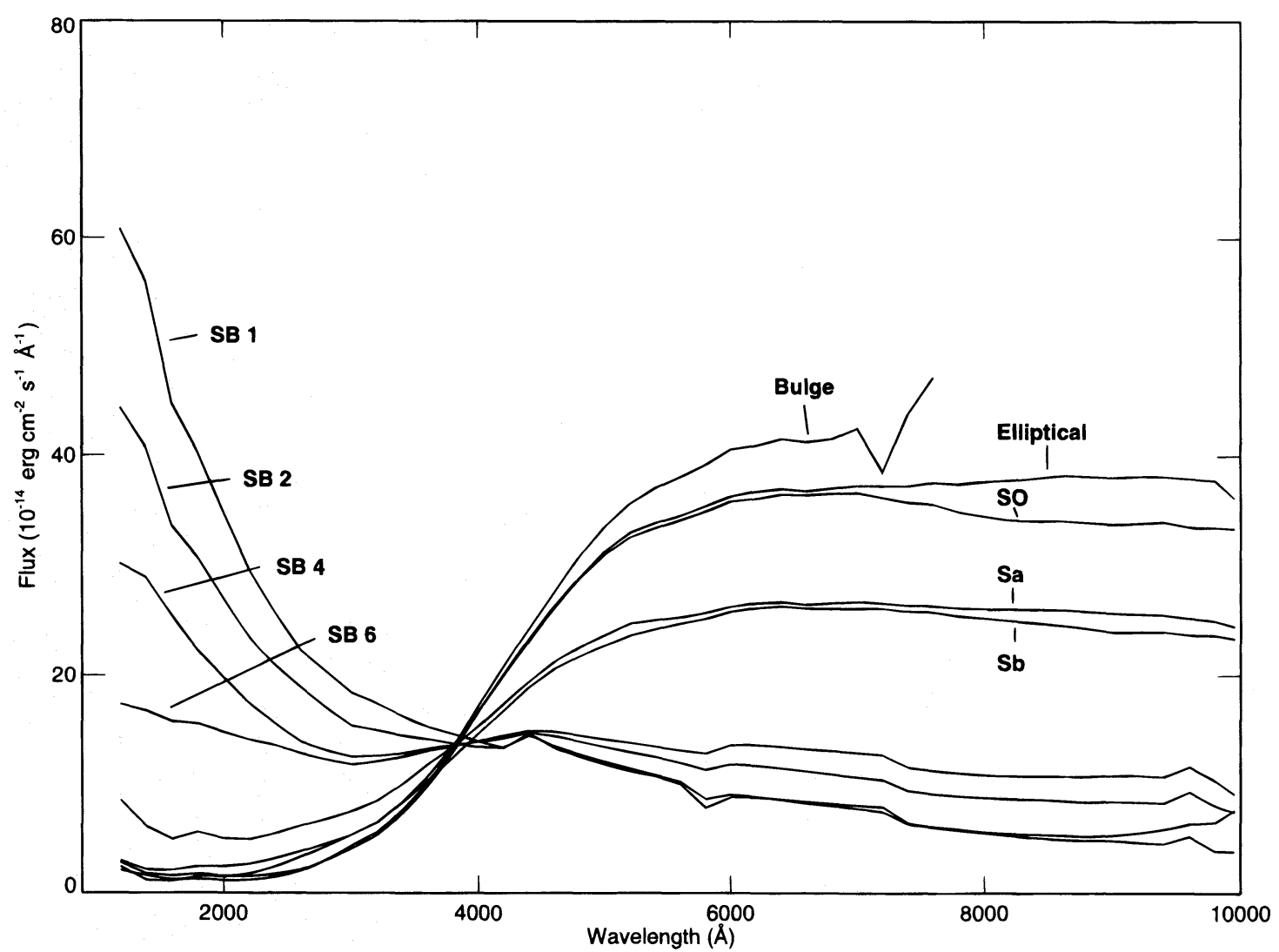

FIG. 1.-The spectra binned in $200 \AA$ bins are shown between 1200 and $10,000 \AA$, normalized at $4000 \AA$ for the bulge, elliptical, S0, Sa, Sb, and starburst galaxies with varying dust content. The starburst galaxy templates are grouped according to the mean color excess value $E(B-V)$ and are labeled as SB1 for $E(B-V)<0.10$; SB2 for $0.11 \leq E(B-V) \leq 0.21$; SB4 for $0.39 \leq E(B-V) \leq 0.50$; and SB6 for $0.61 \leq E(B-V) \leq 0.70$.

in flux level. The UV spectra are averaged according the same procedure adopted for the starburst spectra.

The templates are presented in Figure 1, normalized to the flux at $4000 \AA$. This normalization is chosen to emphasize the spectral difference between the morphological types. The individual templates and their $1 \sigma$ standard deviations are shown in Figures $2 a-2 l$. Because the spectra have been taken in large apertures, the strong night skyline around $5500 \AA$ is difficult to remove and has been masked in most of the plots.

\subsection{Digital Distribution of the Template}

The templates, and the multiwavelength spectra of the individual galaxies on which they were based, will be available in digital form through the National Space Science Data Center (NSSDC), via ftp at host ftp.stsci.edu, and eventually through the Data Archive and Distribution System (DADS) at STScI. The files will be available both in FITS format and in ASCII. Electronic mail requests for the NSSDC can be directed to archives@ndadsa.gsfc.nasa.gov, or via nssdc.gsfc.nasa.gov. The host ftp.stsci.edu (130.167.1.2) will contain the data in the directory catalogs/ spectra.

\section{TEMPLATE APPLICATIONS}

\subsection{General Properties}

The early-type galaxies (E, bulge, and S0) have the reddest spectra, with the largest increase in flux from the $\mathrm{UV}$ to the optical (see Fig. 1). The Sa and Sb galaxies have a slightly weaker increase, while the spectra of the Sc galaxies tend to be rather flat. The sequence of spectral shapes from $\mathrm{E}$ to Sc mirrors the increasing contribution of the disk stellar population relative to the bulge population going from early to late Hubble types. Starburst galaxies become increasingly bluer, with a spectrum that rises toward shorter wavelengths, as the dust obscuration, i.e., $E(B-V)$, decreases.

The E, bulge, S0, Sa, and Sb galaxies have very similar spectral shapes and absorption features for $\lambda>5000 \AA$, and would be rather indistinguishable from their broadband optical and near-IR colors (cf. Kennicutt 1992). The E and S0 templates have similar UV spectra and $4000 \AA$ Aalmer discontinuities. The strength of the $4000 \AA$ discontinuity decreases from the $\mathrm{E}$ and $\mathrm{S} 0$ templates to the $\mathrm{Sa}$ and the $\mathrm{Sb}$ templates.

Our templates are made of galaxies with a variety of physical properties (cf. Table 1), which is important for building representative "galaxy spectral templates." However, the galaxies also show a variety of sizes, and the $I U E$ aperture, although it is the largest available for UV spectroscopy, samples only their inner $15^{\prime \prime}$ region. The aperture/galaxy size mismatch is not a problem for the starburst templates, because their integrated spectral characteristics are dominated by the central burst of star formation (cf. Calzetti et al. 1995). The mismatch is not likely to be a problem for the early Hubble types (E, bulge, and S0), because their integrated emission is dominated by the bulge stellar population, and the inner regions can be considered representative of the galaxies as a whole.

A direct comparison of our templates with Kennicutt's 

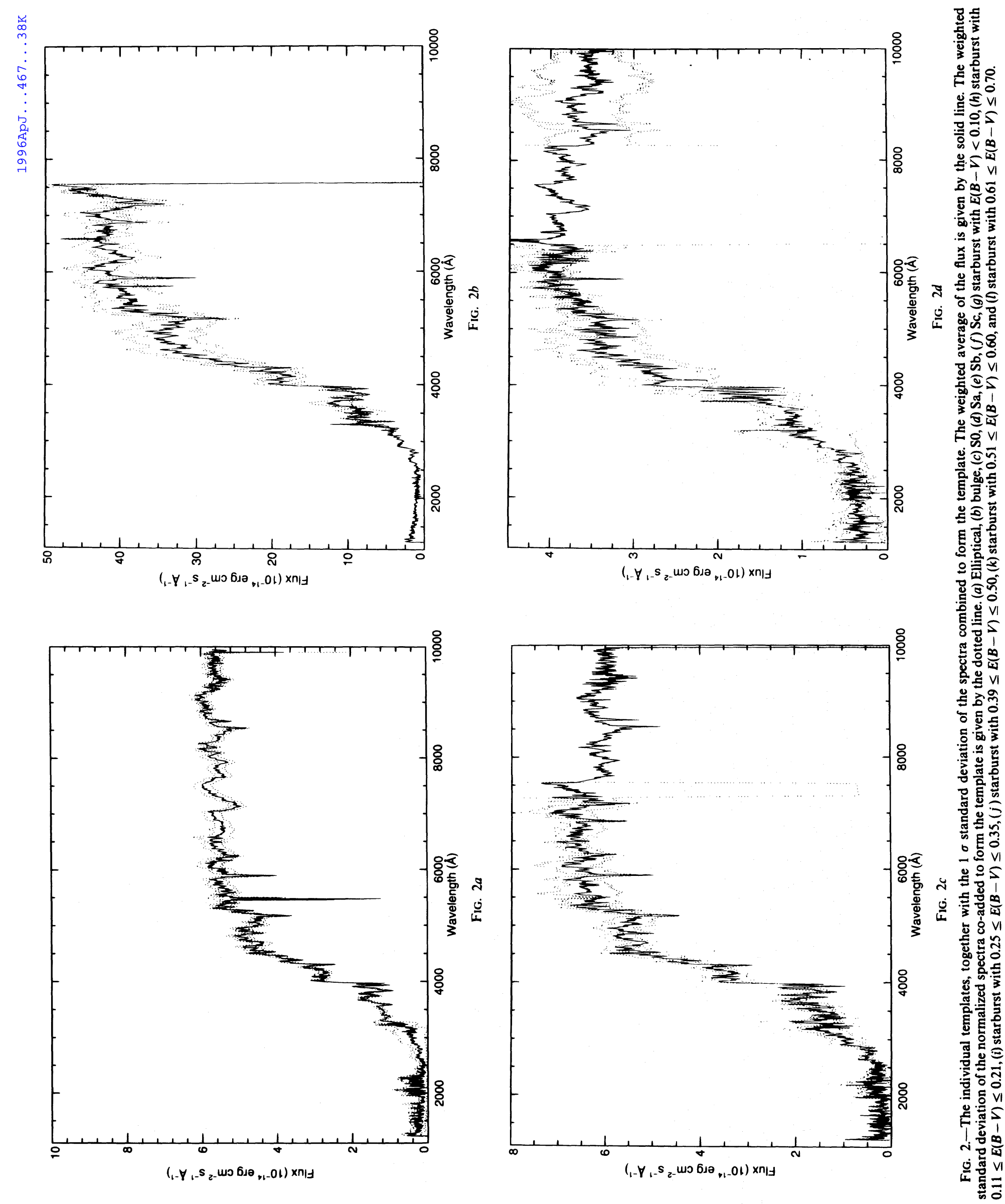

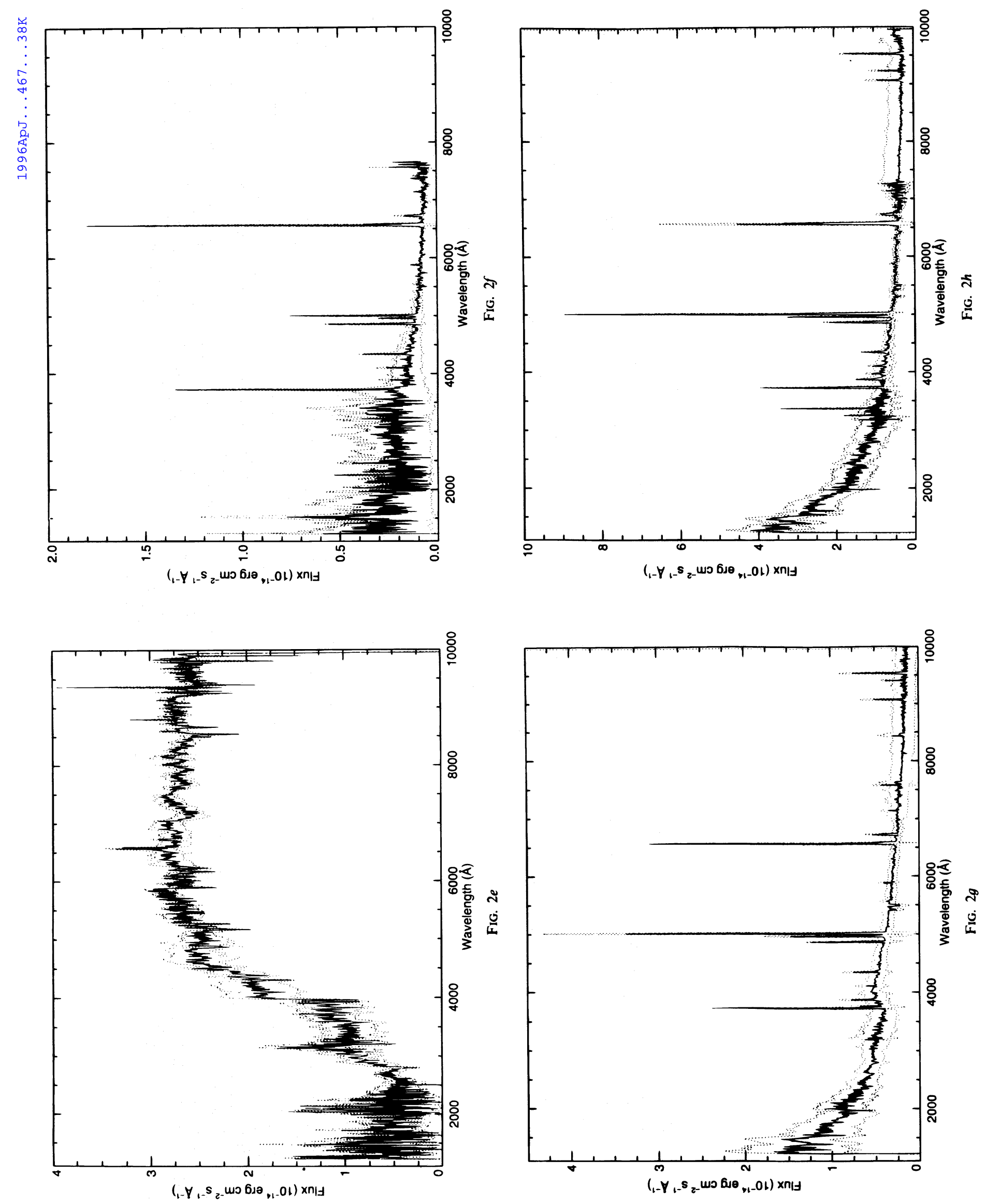

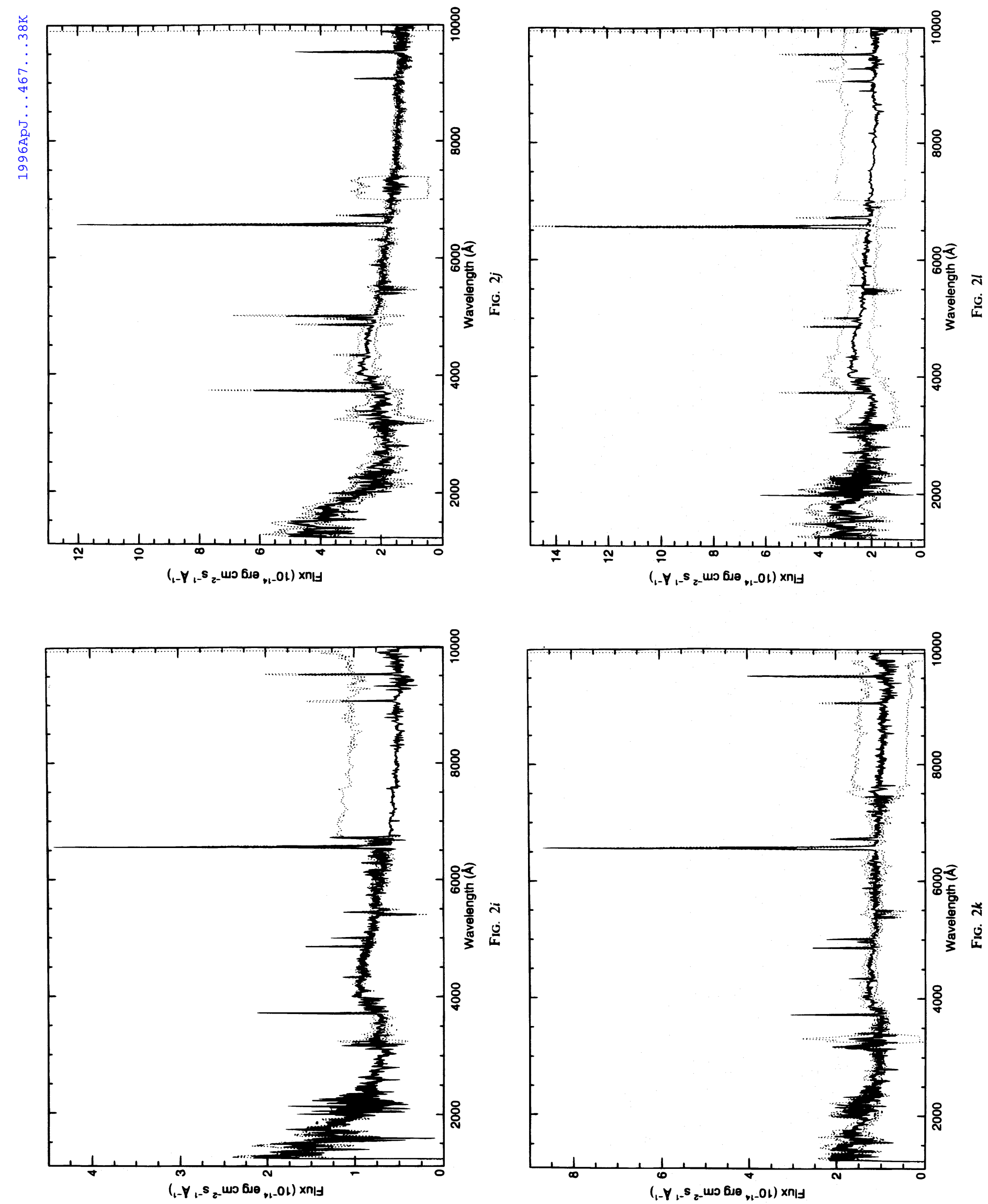
(1992) large aperture $\left(\sim 90^{\prime \prime}\right)$ optical spectra of quiescent galaxies shows that our $\mathrm{E}, \mathrm{S} 0, \mathrm{Sa}$, and Sc templates are representative of the galaxies global properties. However, our $\mathrm{Sb}$ template underestimates the younger disk stellar population relative to the old bulge population. This conclusion is based mostly on the comparison between the emission-line strengths: our $\mathrm{Sb}$ template shows weaker nebular emission lines than Kennicutt's $\mathrm{Sb}$ spectra, meaning that the template underrepresents the youngest stellar populations, which dominate in the external galaxy regions.

The main selection criterion for the galaxies in the sample is their detectability at UV wavelengths, and therefore the sample of quiescent galaxies, which have weak UV fluxes, includes only bright, local galaxies, while the sample of starburst galaxies, which have a large range in UV brightness, includes galaxies up to redshift $z \sim 0.03$. Therefore, the templates of quiescent galaxies give the spectral shapes as a function of galaxy type but give little information on spectral shape as a function of luminosity (cf. next section).

\subsection{Identification of High-Redshift Galaxies}

The identification of high-redshift galaxies is hampered by the general lack of emission lines in the galaxy spectra for deriving secure redshifts. The quiescent galaxies are distinguished mainly by the difference in their UV and optical continuum shape. Only the starburst galaxies show emission lines, and those emission lines appear only in the optical region. The shortest wavelength emission line in starburst galaxies is [O III] $\lambda 3727$, which is already shifted into the $I$ band at a redshift of a little more than 1.0 .

Can colors be used to identify galaxies and derive their redshift? Not easily. If the redshift is known, for example, in the case of a galaxy discovered through narrowband imaging in the redshifted light of $\mathrm{Ly} \alpha$ (for a review see Giavalisco, Macchetto, \& Sparks 1994), the observed color will distinguish between morphological types, as can be seen in Figures $3 a$ and $3 b$. However, if both the redshift and the morphological type must be determined simultaneously, the problem is more difficult. The slopes of the optical continuum longward of $5000 \AA$ and the slopes of the UV continuum shortward of $3000 \AA$ do not vary substantially between the different morphological types. Thus, the predominant identifying and differentiating feature in the SEDs of the different types of galaxies is their behavior between 3000 and $5000 \AA$, i.e., the $4000 \AA$ Balmer discontinuity. The 4000 $\AA$ discontinuity is the strongest for the bulge and E galaxies and grows progressively weaker for the SO, Sa, and Sb galaxies. In the starburst galaxies, the spectra will show a reversal at $4000 \AA$; at shorter wavelengths, they increase in flux inversely proportional to their dust content (see Calzetti et al. 1994).

A better approach may be to use observed magnitudes directly, in order to derive a robust description of morphological type and redshift of galaxies, as described in detail in Connolly et al. (1995). The templates for the $\mathrm{E}, \mathrm{Sa}, \mathrm{Sb}$, and Sc galaxies are shown in Figure 4, redshifted through the $U$, $B$, and $I$ bandpasses to produce a grid of magnitudes for each template as a function of redshift. The galaxies form a well-defined plane in the $U, B, I$ cube. Given multiple bandpasses of information on a large survey of galaxies, such as will be produced in the Sloan Survey (Gunn \& Knapp 1992), statistical identifications can be made based on a comparison between the data and the templates.
Characterizing the thickness of the plane within the $U, B$, $I$ cube would be useful. In other words, how do the colors depend on luminosity for each morphological type? As discussed above, the galaxies making up the sample have little range in luminosity since the $I U E$ satellite can detect only the brightest, nearby galaxies, so this data set cannot address the spectral dependencies on luminosity.

\subsection{Identifying the Host Galaxies of Quasars}

Our spectral energy distributions can be joined with published data in other wave bands to obtain complete wavelength coverage, in some cases going from the radio to the X-ray of a variety of extragalactic objects. Although the apertures through which such broadband spectra are taken vary from wave band to wave band, the data have been chosen from the literature so as to minimize the difference in aperture size. There are some notable exceptions, such as $I R A S$, which has only a very large aperture. However, there is evidence in Calzetti et al. (1995) that the IRAS and the $I U E$ fluxes of starburst galaxies are well correlated regardless of the large difference in the aperture of the two satellites, implying that the differences in this case may be minimal.

We have produced a plot comparing a normal S0 galaxy with a starburst galaxy, a Seyfert 2 galaxy, a template radioquiet quasar, and a template radio-loud quasar. The SEDs shown in Figure 5 are from Sanders et al. (1989, the radioloud and radio-quiet quasars), from Kinney et al. (1993, the UV spectra of the starburst, Seyfert 2, and S0 galaxies), from Weedman et al. (1981) and Israel \& van der Hulst (1983, the radio and X-ray data on NGC 7714), from Ulvestad \& Wilson (1984), Condon et al. (1982), and Pedlar et al. (1983, the radio data on NGC 1068), from Lawrence \& Elvis (1982, the X-ray data on NGC 1068), from the IRAS PointSource Catalog (the far-IR data for NGC 1068, NGC 1553 , and NGC 7714), from Roche et al. (1991, the near-IR data for NGC 1068 and NGC 7714), from Fabbiano, Kim, \& Trinchieri (1992, X-ray data on NGC 1553), from Subrahmanya \& Harnett (1987, radio data on NGC 1553), and from Sadler (1984, radio data on NGC 1553).

To make a comparison between the SEDs of normal galaxies and galaxies with some activity, the starburst, Seyfert 2 , and S0 galaxies are normalized in the red, at $1.2 \mu \mathrm{m}$, thus normalizing by their old star population. The Seyfert 2 galaxy shows little evidence for a hot star component such as dominates the starburst galaxy in the UV at $\log v=15.5$. However, the spectrum of the Seyfert 2 galaxy flattens out in the UV as the featureless blue continuum begins to dominate over the UV flux from the galaxy. The S0 galaxy shows a steeply decreasing UV flux, indicative of the lack of a hot star component. The X-ray flux of the Seyfert 2 galaxy is dominated by the active nucleus, with a UV-to-X-ray slope similar to that of the radio-loud and the radio-quiet quasars, while the lower relative X-ray flux of the starburst galaxy is most likely dominated by supernovae from the hot star population. The X-ray flux of the So galaxy is likely from a hot gaseous halo and, possibly, binary X-ray sources (Fabbiano 1989; see also Bregman, Hogg, \& Roberts 1995).

In the IRAS bands, the Seyfert 2 galaxy shows strong emission from a hot dust component ( $\approx 500 \mathrm{~K})$, while the starburst galaxy has stronger emission from the warm dust component ( $\approx 40 \mathrm{~K}$; see also Calzetti et al. 1995). The S0 galaxy lacks the UV radiation to heat the dust to temperatures as high as those seen in the starburst galaxy and 


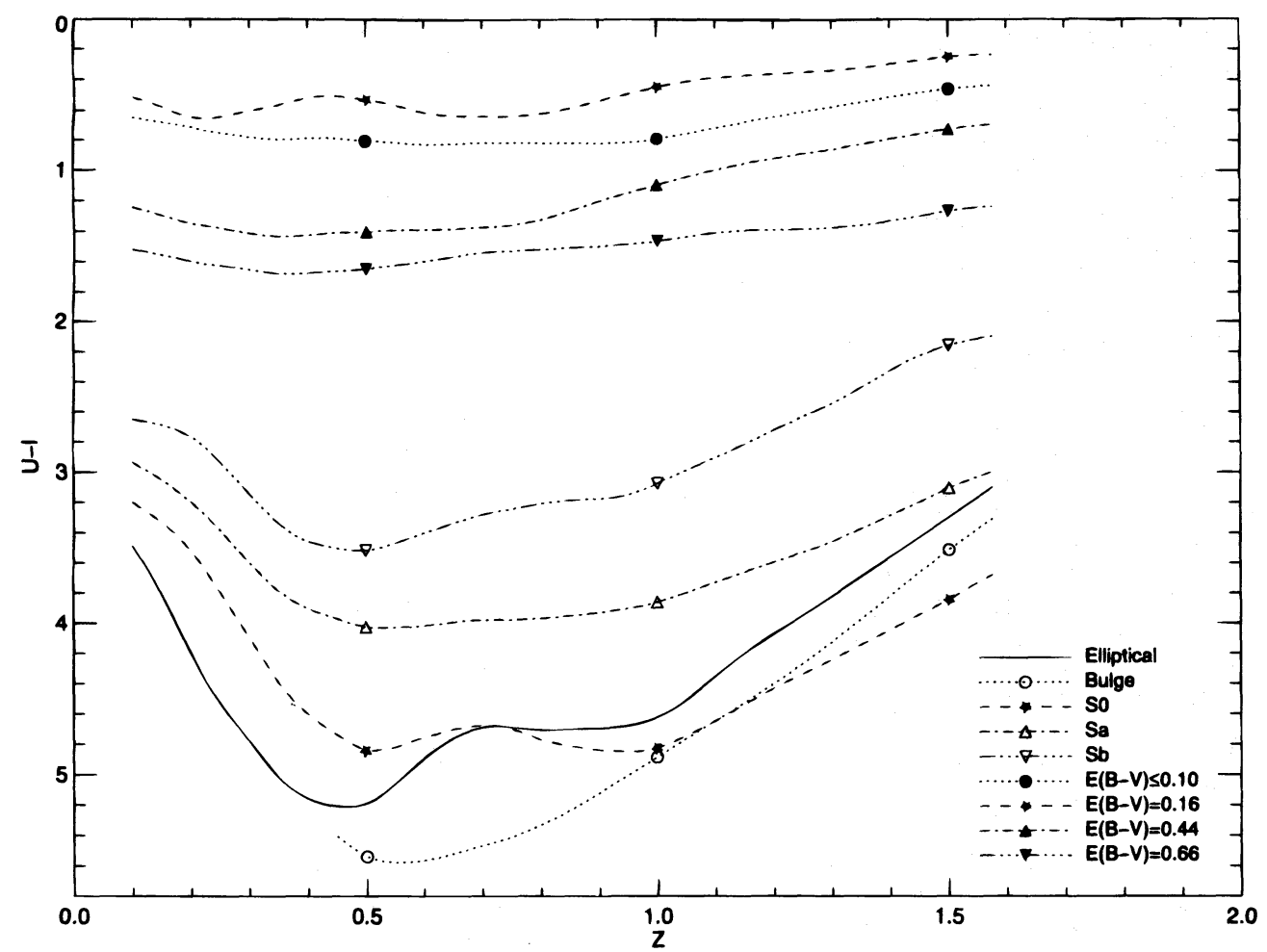

Fig. $3 a$

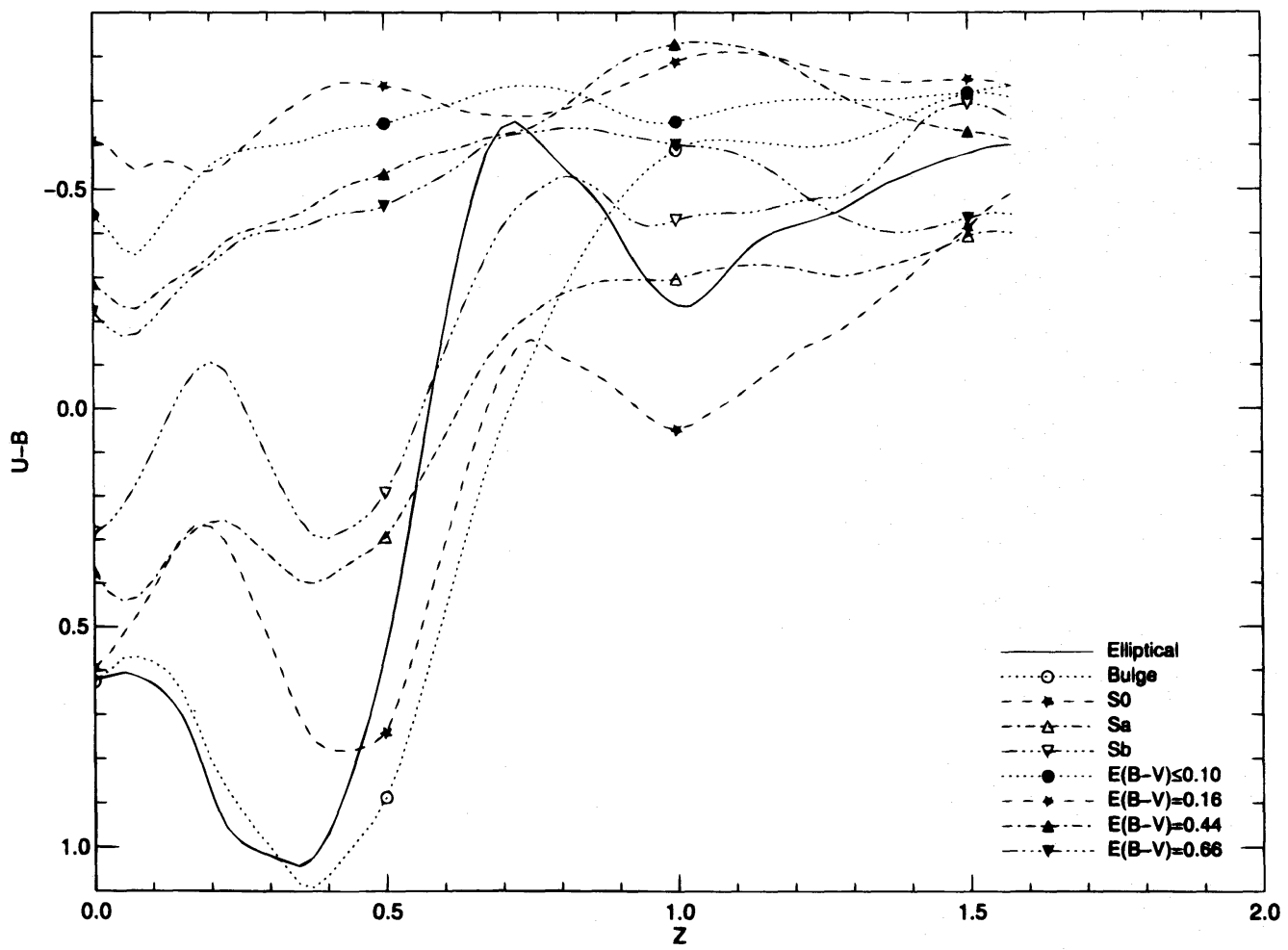

Fig. 3b

Fig. 3. $-(a)$ Color $(U-I)$ vs. redshift for the template galaxies. (b) Color $(U-B)$ vs. redshift for the template galaxies.

therefore has weak emission in the IRAS wave bands.

The choice of the normalization between the galaxies' SEDs seems straightforward; the normalization in the nearIR, where the spectrum is dominated by old stars, simply allows a comparison by the mass of the galaxies. The choice of a normalization between the Seyfert 2 and the quasars is more of a best guess about how to compare the SEDs for the two types of objects. To make the comparison between 


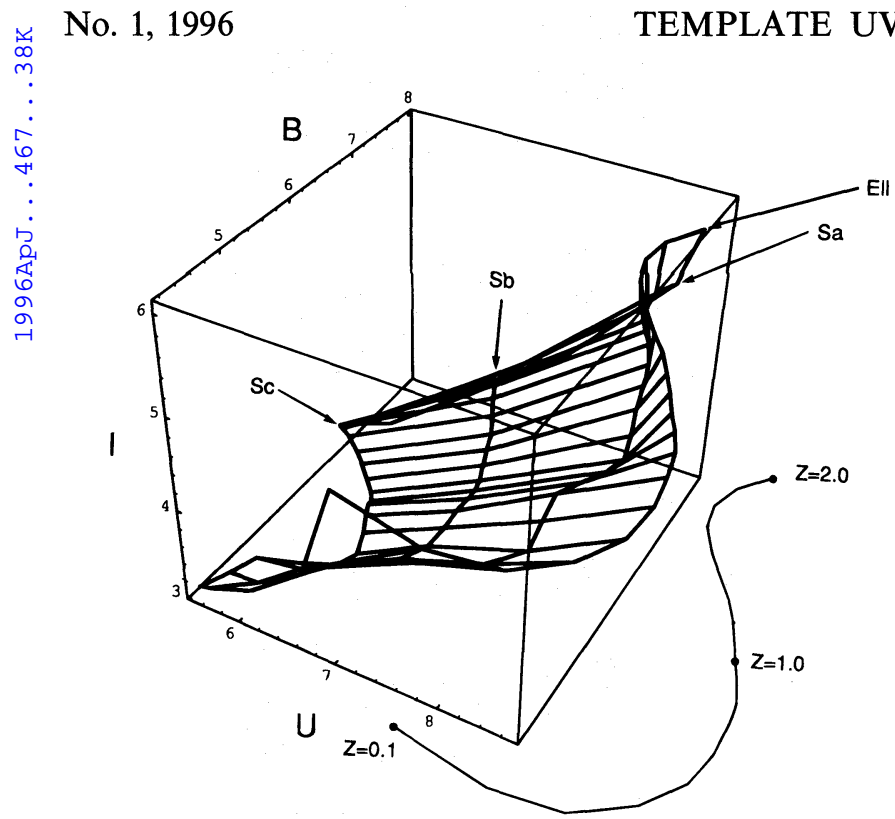

Fig. 4.- $U, B$, and $I$ magnitudes are shown for the normal galaxy templates as a function of redshift, from $z=0$ to $z=2$. The grid shows the magnitudes for the elliptical, $\mathrm{Sa}, \mathrm{Sb}$, and $\mathrm{Sc}$ templates as four lines defining a curved surface. Lines of equal redshift, in increments of 0.1 , are drawn perpendicular to the four lines. The galaxies have been normalized in the red. The curved line to the right of the cube shows the path of the elliptical galaxy template with redshift $z=0.1, z=1.0$, and $z=2.0$ marked.

the Seyfert 2 galaxy, which has regions of its spectrum clearly dominated by the galaxy and regions clearly dominated by its active nucleus, and the quasars, we normalize the spectra at the IRAS $60 \mu \mathrm{m}$ band. Detailed modeling of the dusty torus in active galaxies (Pier \& Krolik 1992) shows that the flux between 60 and $100 \mu \mathrm{m}$ is from the torus and is almost independent of the viewing angle of the torus. So the torus' contribution to the far-IR is isotropic, and a normalization at those far-IR wave bands should be a normalization to the size of the dusty torus. As long as we do not include blazars and BL Lac objects, there should be no further beamed contribution at the far-IR in the active objects (Antonucci 1993). Optical, UV, and X-ray bands are not suitable for the normalization, because they are dominated by a component that is dependent on the viewing angle (Antonucci 1993).

Several conclusions can be drawn from the SEDs of these extragalactic objects. First, the radio emission of the radioquiet quasar is comparable to that of the starburst galaxy. Most likely, the radio emission of radio-quiet quasars is due to the star formation that accompanies activity in the center of the galaxy. Second, in the optical-to-UV region of the SEDs, the galaxies are only a factor of $10-100$ fainter than the quasars. Thus, if the quasar's emission can be diminished by a factor of 100 with observing techniques (for example, by using the coronographic spot on NICMOS on the Hubble Space Telescope), the host galaxy SED should be readily observable. Finally, the red end of the optical spectrum is the best place to observe the host galaxy-before the big blue bump of the quasar begins to dominate the spectrum. However, based on the templates shown in Figure 1 , the $4000 \AA$ region is the region that most distinguishes between different galaxy types. Thus, the red spectral region, where the host galaxy is most readily observable, is not the same as the spectral region $(4000 \AA)$, where the host galaxy is most readily classifiable.

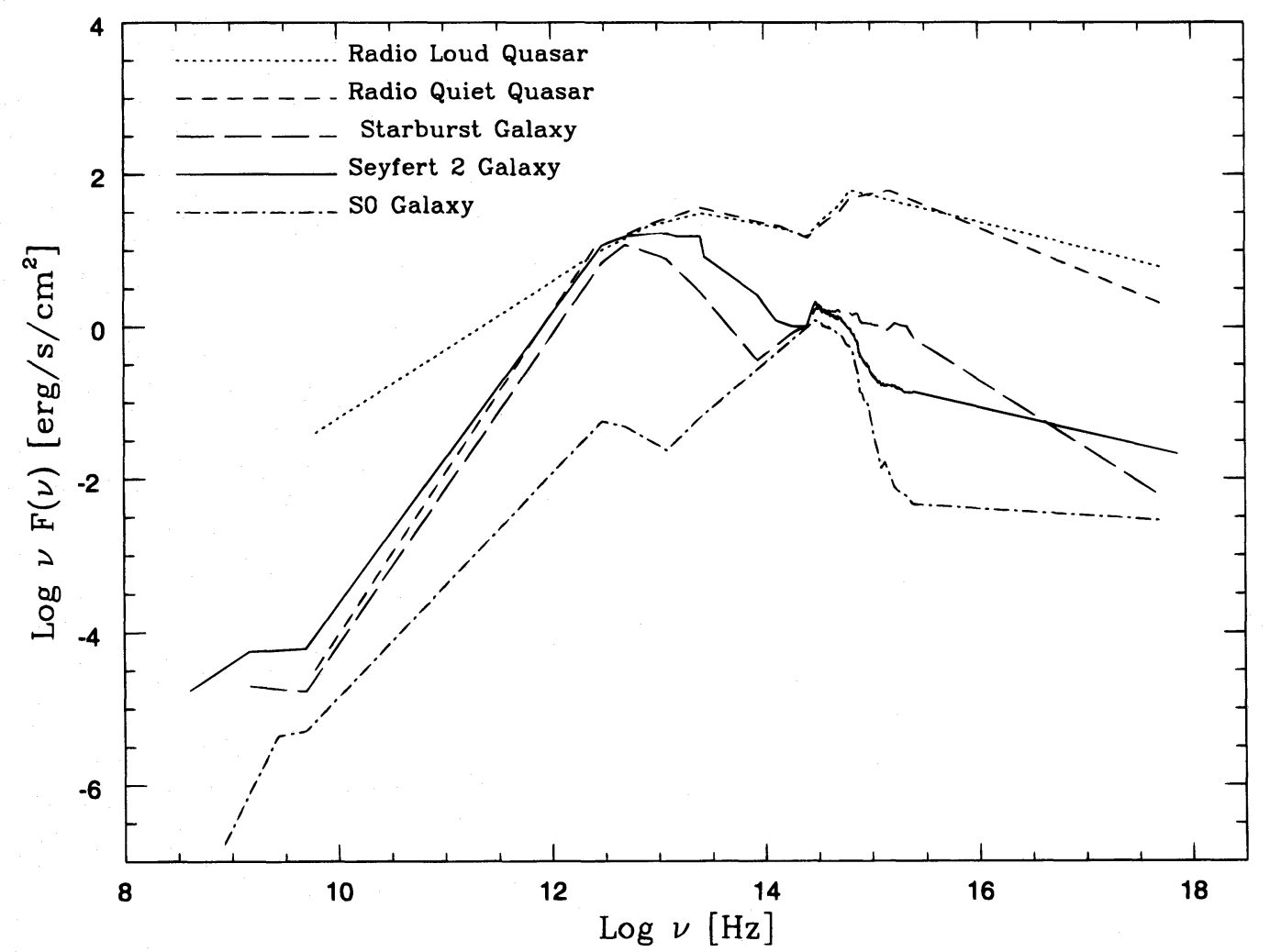

Fig. 5.-SED for the S0 galaxy NGC 1553, for the starburst galaxy NGC 7714, and for the Seyfert 2 galaxy NGC 1068 normalized at $1.2 \mu$ m, overlaid with a template radio-loud quasar and radio-quiet quasar from Sanders et al. (1989). The quasars are normalized to the Seyfert 2 galaxy by the flux in the IRAS band at $60 \mu \mathrm{m}$, a flux that is thought to be isotropic and therefore not dependent on the orientation angle of the active nucleus. 


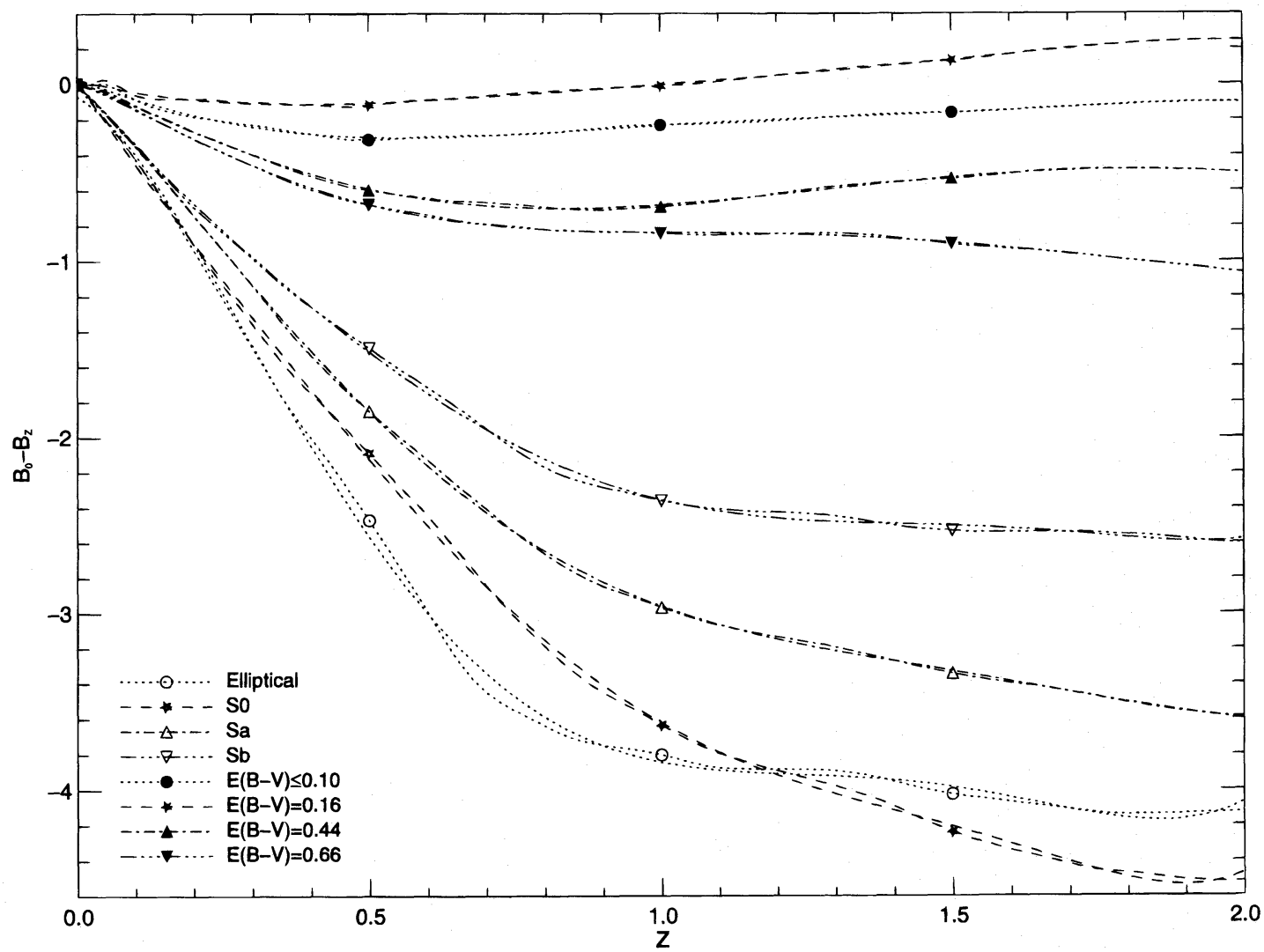

FIG. 6.-K-corrections $[B(z=0)-B(z)]$ as a function of redshift for the template galaxies. The polynomial fits are shown overlaid on the $K$-corrections. The fit parameters are given in Table 3 .

\subsection{K-Corrections and the Faint Galaxies' Number Counts}

The number of faint blue galaxies detected in deep surveys (e.g., Koo \& Kron 1982; Broadhurst, Ellis, \& Shanks 1988) exceeds the number predicted from noevolution models, typically based on the $K$-corrections ${ }^{7}$ of Pence (1976) and Coleman, Wu, \& Weedman (1980). The deviation of the number counts from the no-evolution hypothesis has been used to postulate various theories for the galaxy evolution. Indeed, more drastic processes than the passive evolution of the galaxy stellar populations are required in order to explain the excess in number counts, although the redshift distribution appears to be in remarkable agreement with the no-evolution hypothesis (for a review, see Koo \& Kron 1992).

This section discusses the effect of the $K$-corrections on the expected number counts. While the Pence (1976) and Coleman et al. (1980) $K$-corrections were the best available for many years, as evidenced by their continuous use, the corrections are based on an inhomogeneous sample of UV and optical spectral data of galaxies, in order to reach a redshift of $z=1.4$. Our template spectra match aperture size and morphological type with, typically, five galaxies

\footnotetext{
${ }^{7}$ The commonly used term $K$-correction originated as a reference to the $K$-term by Wirtz (1918), where $K$ stands for the German word for constant, konstante. The $K$-term was a constant offset in redshift applied to "diffuse nebula," which were found to be receding, on average, by a velocity of $656 \mathrm{~km} \mathrm{~s}^{-1}$. The average recession velocity was originally interpreted as local motion.
}

contributing to the UV spectral range and three of the same galaxies contributing to the optical spectral range (see Table 2 ), reaching a redshift of $z=2.0$ for some bandpasses. Thus, these are self-consistent templates that can be used to calculate more accurate $K$-corrections as a function of redshift than previously available. These $K$-corrections can be used for a number of applications, including predictions of the number of faint blue galaxies as a function of magnitude. The $K$-corrections have been fitted to sixth-order polynomials and are shown as a function of redshift in Figure 6. The constants for the fits are given in Table 3. Our $K$-corrections for the bulge and $\mathrm{E} / \mathrm{S} 0$ galaxies are similar to previous $K$-corrections (e.g., King \& Ellis 1985 based on Pence 1976 and Coleman et al. 1980), but we also have a large dispersion of slopes as a function of morphological type, all the way to an almost constant value for the very blue starburst galaxies.

To evaluate the faint blue galaxies' number counts, we invoke assumptions similar to those used by Broadhurst (1989) and start with a mix of galactic morphological types based on the Durham/Anglo-Australian Redshift Survey (DARS, Broadhurst et al. 1988), but with an added starburst component. We assume a mixture of galaxies with $24 \%$ $\mathrm{E} / \mathrm{S} 0,19 \% \mathrm{Sa}, 23 \% \mathrm{Sb}, 29 \% \mathrm{Sc}-\mathrm{Sd}$, and $5 \%$ starbursting galaxies. We assume a Schechter (1976) luminosity function, given as

$$
\phi(L) d L=\phi_{*}\left(\frac{L}{L^{*}}\right)^{\alpha} e^{\left(-L / L^{*}\right)} d L
$$


TABLE 3

Polynomial Fits to $K$-CORRECTIONS:

$B(0)-B(z)=K+A z+B z^{2}+C z^{3}+D z^{4}+E z^{5}+F z^{6}$

\begin{tabular}{|c|c|c|c|c|c|c|c|}
\hline Type & $K$ & $A$ & B & $C$ & $D$ & $E$ & $F$ \\
\hline Elliptical & -0.06592 & -1.94619 & -15.56946 & 25.90334 & -15.81747 & 3.92798 & -0.2762 \\
\hline Bulge $\ldots \ldots \ldots$ & 0.00042 & -4.31986 & -1.21184 & -4.50550 & 11.33809 & -6.92851 & 1.33613 \\
\hline S0 .... & 0.03107 & -5.09886 & 3.87624 & -8.89221 & 11.23979 & -5.85310 & 1.07485 \\
\hline $\mathrm{Sa}$ & 0.01013 & -3.30775 & -3.17835 & 6.07455 & -2.97560 & 0.37363 & 0.04352 \\
\hline $\mathrm{Sb} \ldots$. & 0.00782 & -3.70729 & 2.21292 & -3.65696 & 5.01346 & -2.72938 & 0.50556 \\
\hline Sc $\ldots .$. & -0.00768 & 1.32313 & 0.58448 & -16.14859 & 22.05992 & -10.98042 & 1.89501 \\
\hline SB1 & 0.02797 & -1.18577 & 0.62820 & 1.96862 & -2.81187 & 1.37572 & -0.23526 \\
\hline SB2 & 0.02841 & -0.89573 & 1.87564 & -1.46486 & 0.45232 & 0.03481 & -0.03324 \\
\hline SB3 & 0.04050 & -2.34058 & 5.26435 & -5.52641 & 3.00158 & -0.8093 & 0.08468 \\
\hline SB4 & 0.02050 & -1.36718 & -0.86146 & 3.48411 & -2.76431 & 0.92692 & -0.11951 \\
\hline SB5 .. & 0.00632 & -1.38520 & 0.79897 & 1.32753 & -1.91255 & 0.83166 & -0.12048 \\
\hline SB6 .... & 0.01428 & -1.44780 & -1.65970 & 5.62661 & -4.96270 & 1.84530 & -0.25320 \\
\hline
\end{tabular}

NotES.-SB1 refers to starburst galaxies with color excess $E(B-V) \leq 0.1$. SB2 refers to starburst galaxies with color excess $0.11 \leq E(B-V) \leq 0.21$. SB3 refers to starburst galaxies with color excess $0.25 \leq E(B-V) \leq 0.35$. SB4 refers to starburst galaxies with color excess $0.39 \leq E(B-V) \leq 0.50$. SB5 refers to starburst galaxies with color excess $0.51 \leq E(B-V) \leq 0.60$. SB6 refers to starburst galaxies with color excess $0.61 \leq E(B-V) \leq 0.70$.

where $\alpha=-1.25$. Following the methodology of Broadhurst (1989), we use the mean absolute $B_{j}$ magnitude for the galaxies in the DARS to set $M^{*}=-2.5 \log L^{*}+$ const for each galaxy type:

$$
\begin{array}{ll}
\mathrm{E} / \mathrm{S} 0: & M^{*}=-21.6+5 * \log \left(H_{0} / 100 .\right), \\
\mathrm{Sa} / \mathrm{Sb}: & M^{*}=-21.15+5 * \log \left(H_{0} / 100 .\right), \\
\mathrm{Sa} / \mathrm{Im}: & M^{*}=-20.85+5 * \log \left(H_{0} / 100 .\right), \\
\text { starburst: } & M^{*}=-19.65+5 * \log \left(H_{0} / 100 .\right),
\end{array}
$$

and we use our derived $K$-corrections to predict the number counts. The number counts as a function of the apparent magnitude $m$ are given by

$$
\begin{aligned}
N(m)= & 4 \pi\left(\frac{c}{H_{0}}\right)^{3} \\
& \times \int_{0}^{\infty} \frac{\left[q_{0} z+\left(q_{0}-1\right)\left(\sqrt{1+2 q_{0} z}-1\right)\right]^{2}}{q_{0}^{4}(1+\mathrm{z})^{2} \sqrt{1+2 q_{0} z}} d z \\
& \times \int_{L(z) / L^{*}} \phi(L) d L,
\end{aligned}
$$

with

$$
\frac{L(z)}{L^{*}}=\left[\frac{D(z)}{D\left(z^{*}\right)}\right]^{2} 10^{0.4\left[k(z)-k\left(z^{*}\right)\right]},
$$

where $k(z)$ is the $K$-correction.

Our prediction of number counts in the case of no galaxy evolution, shown as a solid line in Figure 7, underpredicts the observed number counts as reported in Broadhurst et al. (1988). Our predictions are practically indistinguishable from those based on the Pence (1976) data (Fig. 7, dashed line) and show that the number counts are not greatly sensitive to variations in the $K$-corrections (see also King \& Ellis 1985).

As a simple experiment, we also calculate a prediction based on the hypothetical galaxy mixture made up of $100 \%$ mildly reddened starburst galaxies. We find that a population made up entirely of starbursting galaxies with reddening $E(B-V) \sim 0.3$, given by the dot-dashed line in Figure 7 , is compatible with the number counts.

\subsection{Comparison of Templates with Stellar Synthesis Models}

How do the observed templates compare with the SEDs computed in spectral synthesis programs? We make comparisons here for the populations representing the extremes in ages: the elliptical galaxy and the bluest starburst galaxy.

In Figure 8, we overlay the template for the elliptical galaxy (solid line) with the best matching synthetic spectrum (dotted line) based on the Bruzual \& Charlot (1993) code. We use the model of a single instantaneous burst with an age of 16 Gyr. The spectra have been normalized at $4000 \AA$. The model and the template show similar features in general, with the size of the $4000 \AA$ discontinuity, the most marked feature in the template, being well matched. There is some mismatch at the red end of the spectrum, which may

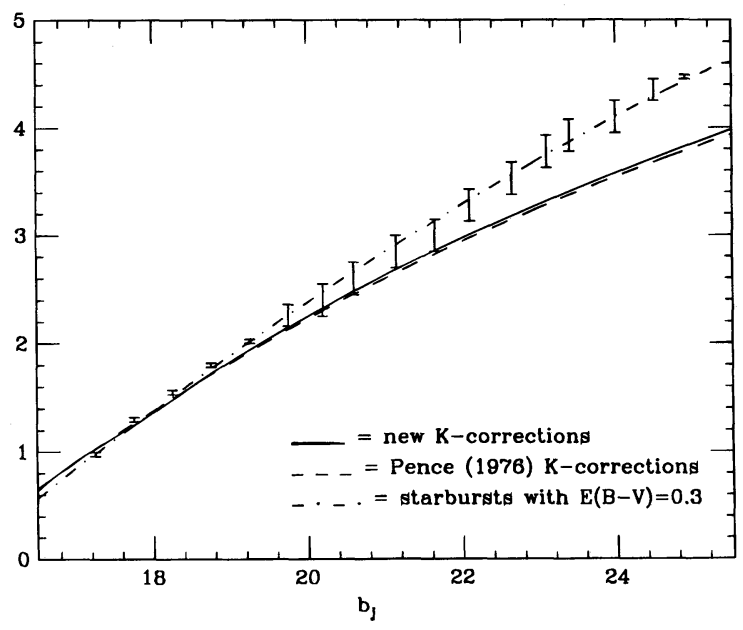

Fig. 7.-Prediction for number counts for the no-evolution galaxy model compared with the data of Broadhurst et al. (1988) as a function of the blue magnitude $B_{j}$. Data are given with $1 \sigma$ error bars. Prediction based on our $K$-corrections are given by the solid line. Predictions based on Pence (1976) $K$-corrections are given by the dashed line. For comparison, a prediction based on a highly unphysical universe made up completely of starbursts with low dust content is given by the dot-dashed line. 


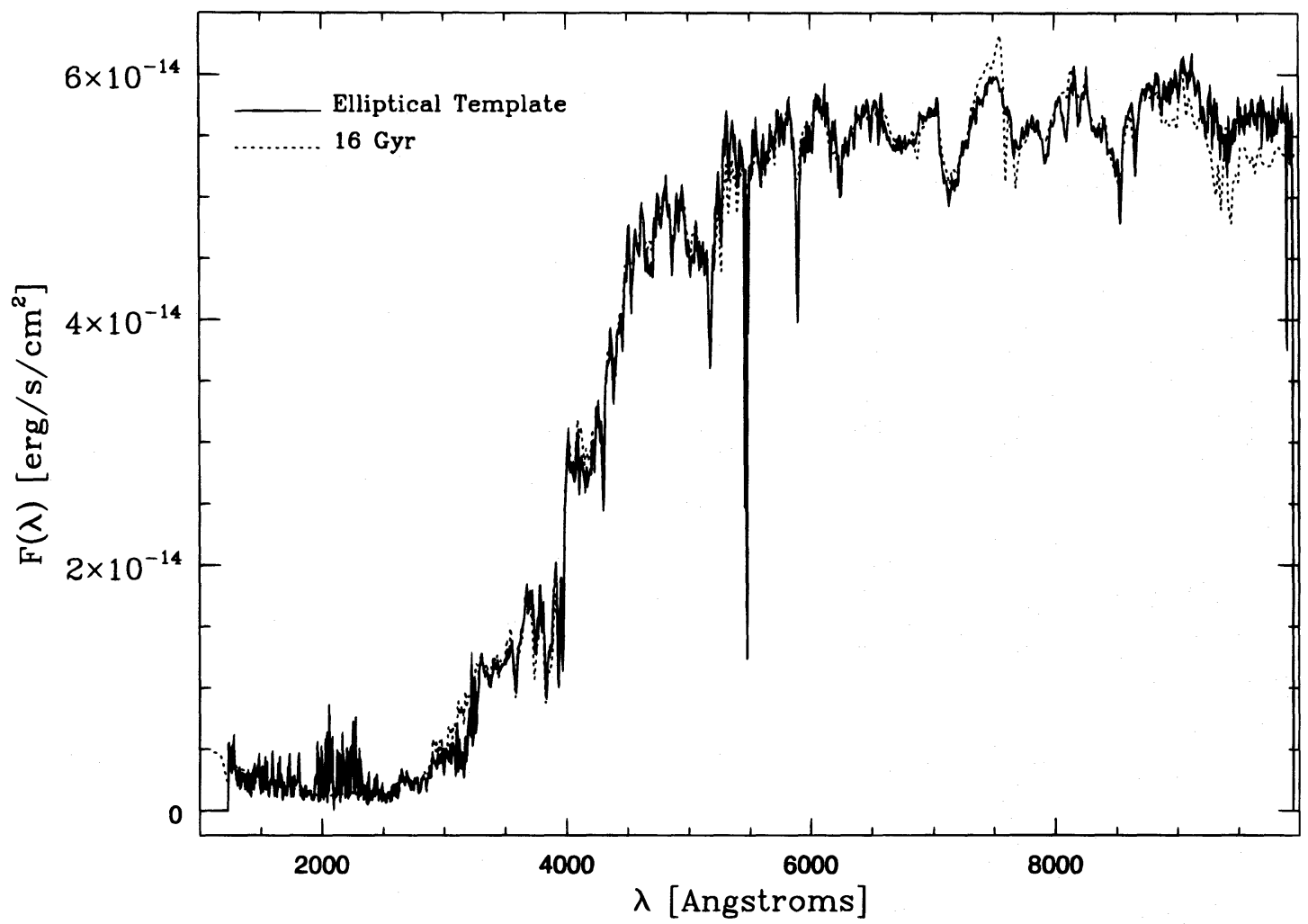

FIG. 8.-The elliptical template (solid line) is plotted with a synthetic spectrum overlaid (dotted line). The spectra are normalized at $4000 \AA$. The synthetic spectrum is based on a single burst model of age $16 \mathrm{Gyr}$.

be attributed to the ongoing developments in understanding the properties of the low end of the initial mass function (Charlot, Worthey, \& Bressan 1996).

Figure 9 shows our starburst template for the case $E(B-V)=0.0$ (solid line) overlaid with a synthetic spectrum (dashed line) based on a Bruzual \& Charlot (1993) model using continuous star formation and an age of $7.2 \times 10^{8} \mathrm{yr}$, and normalized at $4000 \AA$ A. Since Bruzual \& Charlot models to not include nebular emission, the emission lines are lacking in the synthetic spectrum. The continuum match is nonetheless fairly good in the optical and in the long-wavelength end of the UV. The major difference between the two spectra is at wavelengths shorter than 2000 $\AA$, where the template is markedly redder than the synthetic spectrum. The most likely explanation for the difference is that the template spectrum includes star-forming regions of different ages and different dust content, while the synthetic spectrum includes only one dust-free region undergoing continuous star formation (cf. Leitherer \& Heckman 1995). However, for actual comparisons with high-redshift galaxies, the observed templates may be a more realistic version of a "starburst galaxy" than the models.

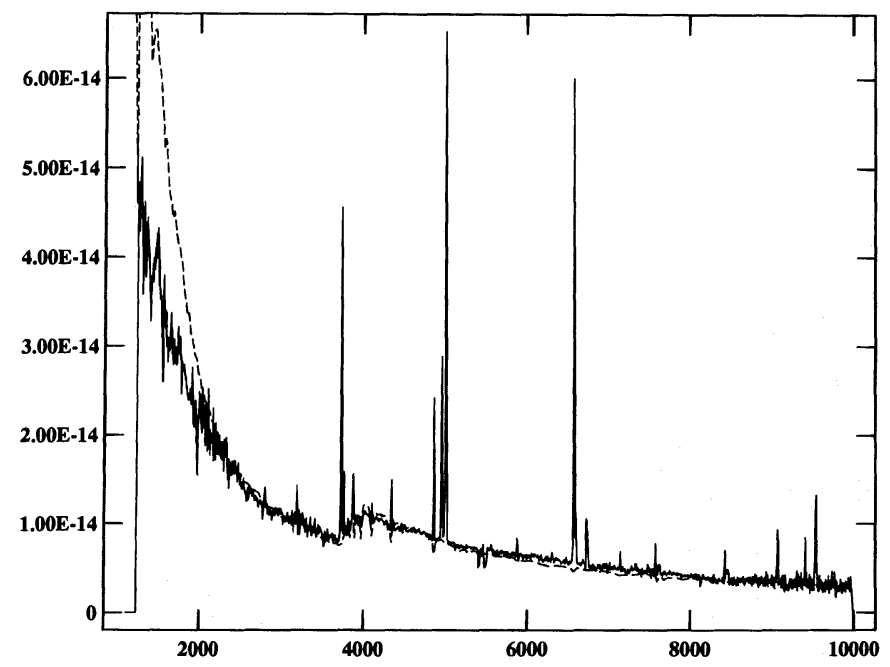

FIG. 9.-The template for the starburst galaxy (solid line) with $E(B-V)=0.0$ is plotted with a synthetic spectrum overlaid (dashed line). The spectra are normalized at $4000 \AA$. The synthetic spectrum is based on a continuous star formation model of age $7.2 \times 10^{8} \mathrm{yr}$. 


\section{CONCLUSIONS}

We present template UV-optical spectra of quiescent and starburst galaxies, from a combination of IUE data and of optical data with an aperture size matched to the IUE. The templates are built according to morphological type for the quiescent galaxies and according to color excess for the starburst galaxies (cf. Calzetti et al. 1994). Comparison of our templates with Kennicutt's (1992) large-aperture optical spectra suggests that, despite our relatively small observational apertures, the galaxy stellar populations are well represented in the elliptical, S0, Sa, and Sc templates. The relatively young disk stars are underrepresented relative to the old bulge population in our Sb template. Starburst galaxies are dominated by the central burst of star formation, which is well sampled by our spectra.

Analysis of galaxy colors shows that colors do not generally help to define a detailed morphological classification of a galaxy or to determine its redshift. However, the magnitudes of the galaxies in multiple wave bands do separate the galaxies both by morphological type and by redshift, so that three-dimensional "magnitude cubes" should provide a useful tool in identifying distant galaxies (Connolly et al. 1995).

The spectra of an S0 galaxy, a starburst galaxy, and a Seyfert 2 galaxy are joined with data from the literature to form multifrequency spectra spanning the range from radio to X-ray. These spectra are displayed together with template active galaxies, allowing a comparison over a wide spectral range. In addition, the reddest (elliptical) template and the bluest ("dust-free" starburst) template are com- pared with stellar synthesis models. There is a general satisfactory agreement between models and templates; the major difference is in the far-UV side of the starburst spectra and can be attributed to the presence of more than one region of star formation contributing to the integrated emission.

Template spectra are used to calculate $K$-corrections for galaxies as a function of morphological type and redshift, up to $z \simeq 2$. Despite the fact that these $K$-corrections are a clear improvement over previous determinations (cf. King \& Ellis 1985; Broadhurst et al. 1988), the blue galaxy number counts they predict in the case of no-evolution are not very different from the number counts predicted by previous $K$-corrections. Improved $K$-corrections do not help resolve the anomalously high number of faint blue galaxies detected by deep surveys (cf. King \& Ellis 1985).

The authors thank Pete Challis, Christine Schwartz, and Cindy Taylor for help with observations and data reduction. The authors acknowledge support from NASA grants NAG 5-1143, NAG 5-1675, and NAGW-3757, and from the STScI Directors Research Fund. T. S. B. and H. R.S acknowledge support from the Brazilian Institutions CNPq, CAPES, and FAPERGS. T. S. B. would like to thank UPD and the Directors Research Fund for support for a collaborative visit to STScI.

This research has made use of the NASA/IPAC Extragalactic Database (NED), which is operated by the Jet Propulsion Laboratory, Caltech, under contract with the National Aeronautics and Space Administration.

\section{APPENDIX}

We have derived color-color and color-versus-redshift diagrams using the standard Johnson bandpasses $U, B, V, R$, and $I$, shown as response versus $\log v$ in Figure 10, and the bandpasses developed by Steidel \& Hamilton $(1992,1993)$ specifically for searching for high-redshift galaxies $\left(U_{n}, G\right.$, and $\mathscr{R}$, shown in Fig. 11). For comparison purposes, the energy distributions of the quiescent galaxy templates are plotted in Figure 12, and the energy distributions of the starburst templates are plotted in Figure 13 as $v F_{v}$ versus $\log v$. The quiescent galaxies have only one noticeable feature, the strong $4000 \AA$ discontinuity, while the starburst galaxies show a slight increase in flux across the $4000 \AA$ discontinuity.

Table A1 lists the template fluxes, normalized by averaging between 3800 and $4200 \AA$ to the bulge spectrum for comparison between the templates. The table lists the mean in each $200 \AA$ bin in units of $1 \times 10^{-14} \mathrm{ergs} \mathrm{cm}^{-2} \mathrm{~s}^{-1} \AA^{-1}$, and the standard deviation in the fluxes in the corresponding bin in the same units.

The color-versus-redshift diagrams and color-versus-color diagrams for the different morphological types at different redshift are shown in Figure 14. 


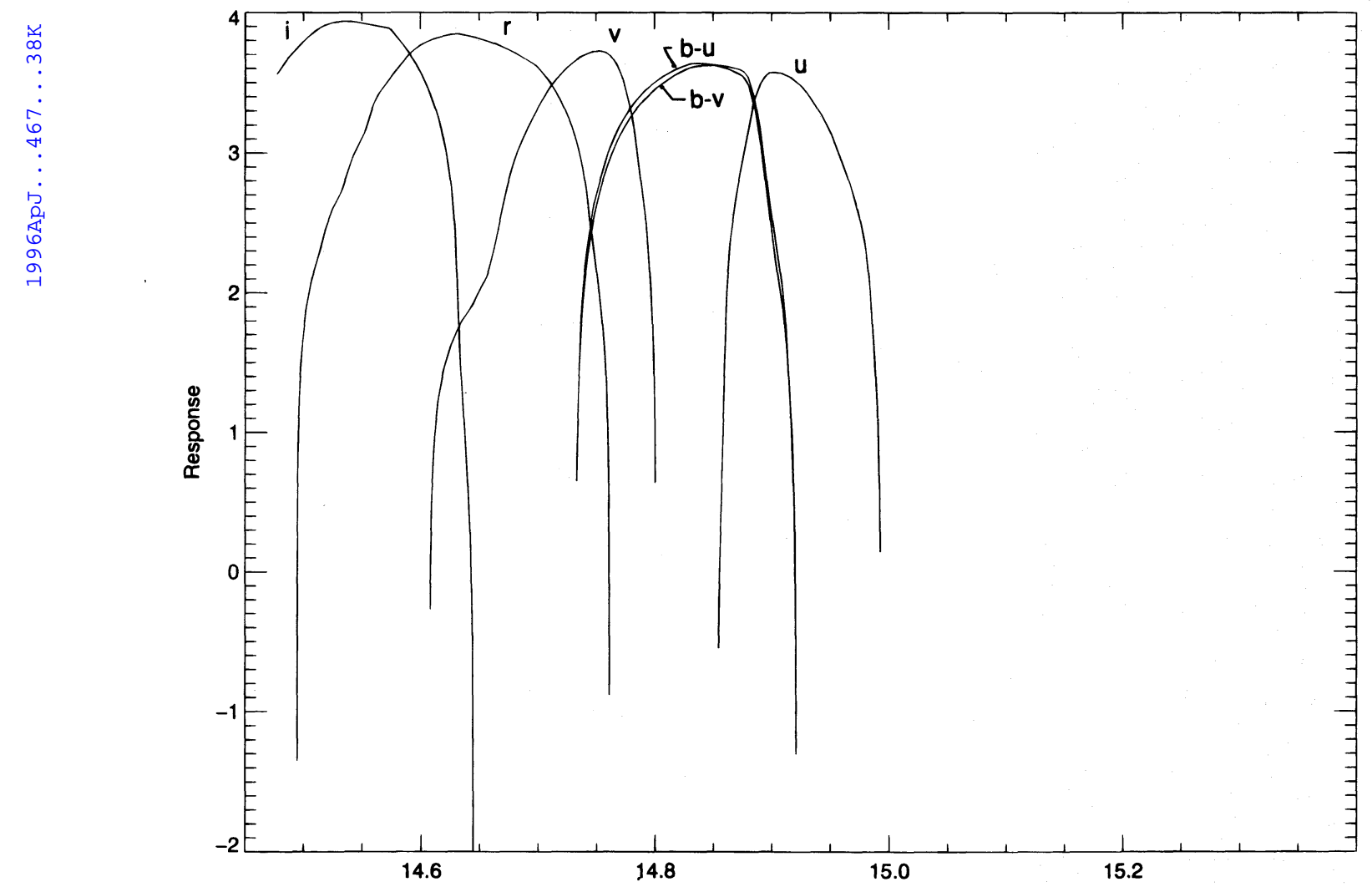

FIG. 10.-Standard bandpasses $U, B, V, R$, and $I$ are shown with the bandpass response plotted vs. $\log v$

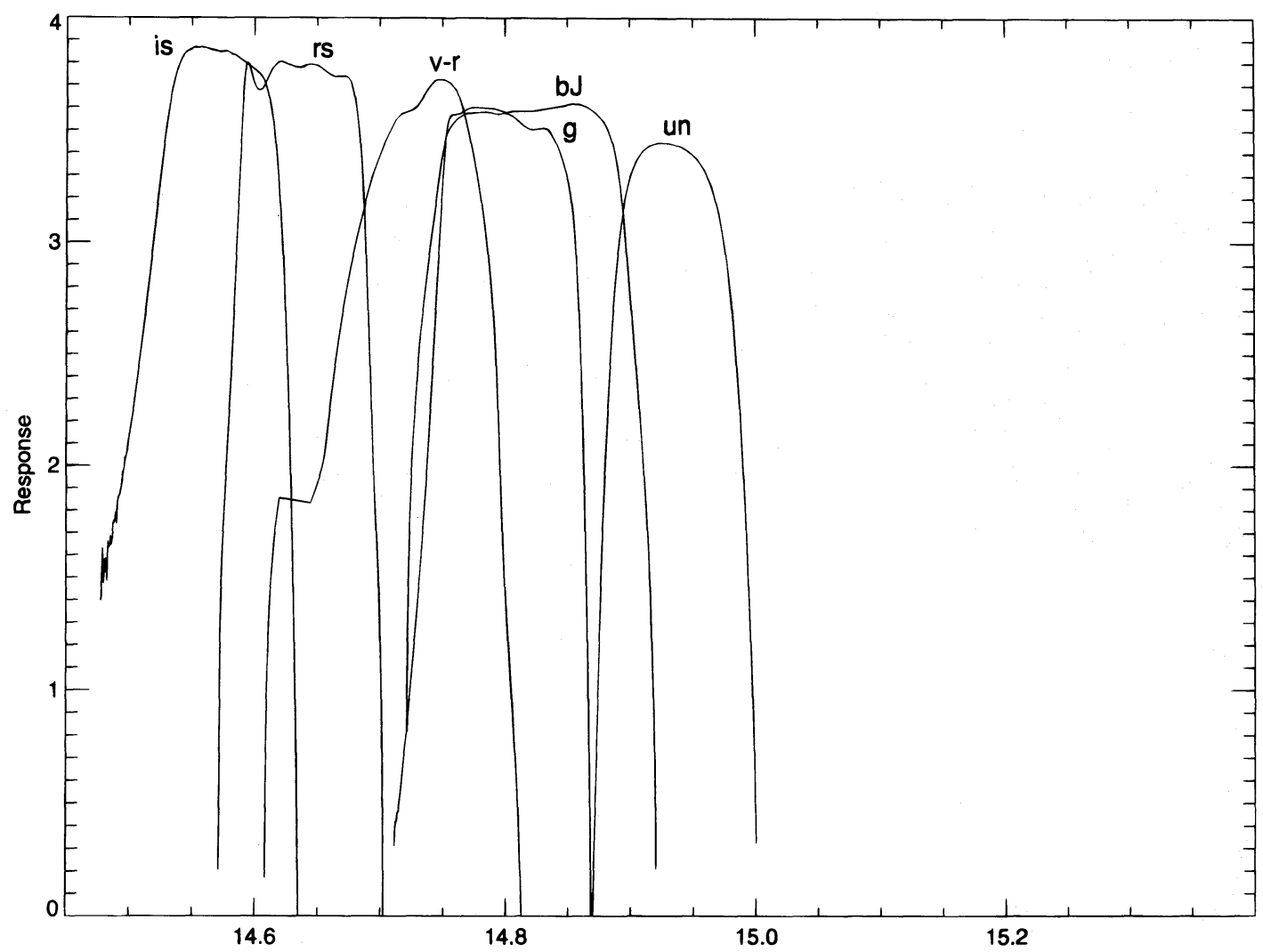

Fig. 11. - Special bandpasses developed by Steidel \& Hamilton $(1992,1993) . U_{n}, G$, and $\mathscr{R}$ are shown with the bandpass response plotted vs. log $v$. 


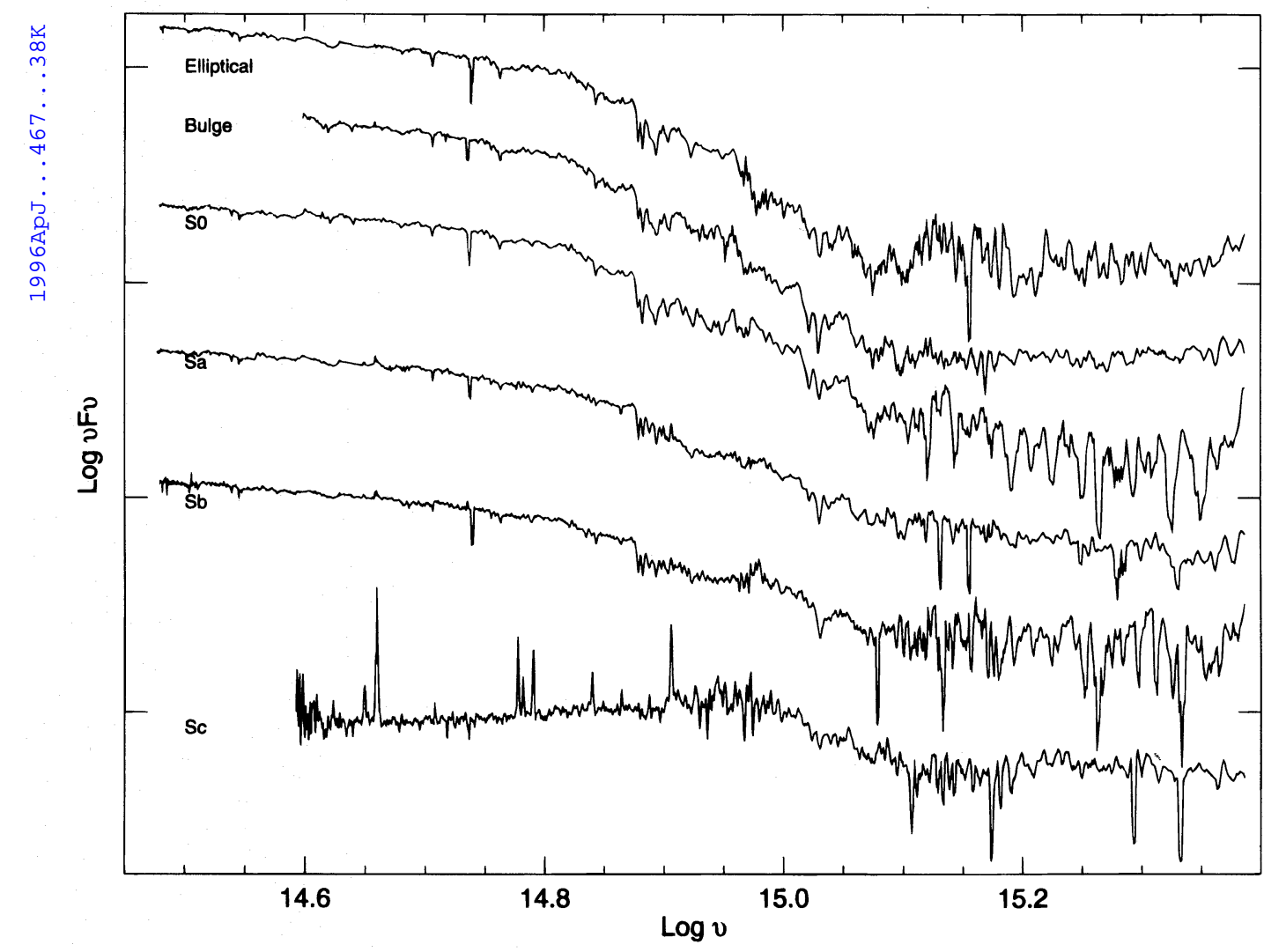

FIG. 12.-Templates of normal galaxies plotted in the same units as Figs. 10 and 11

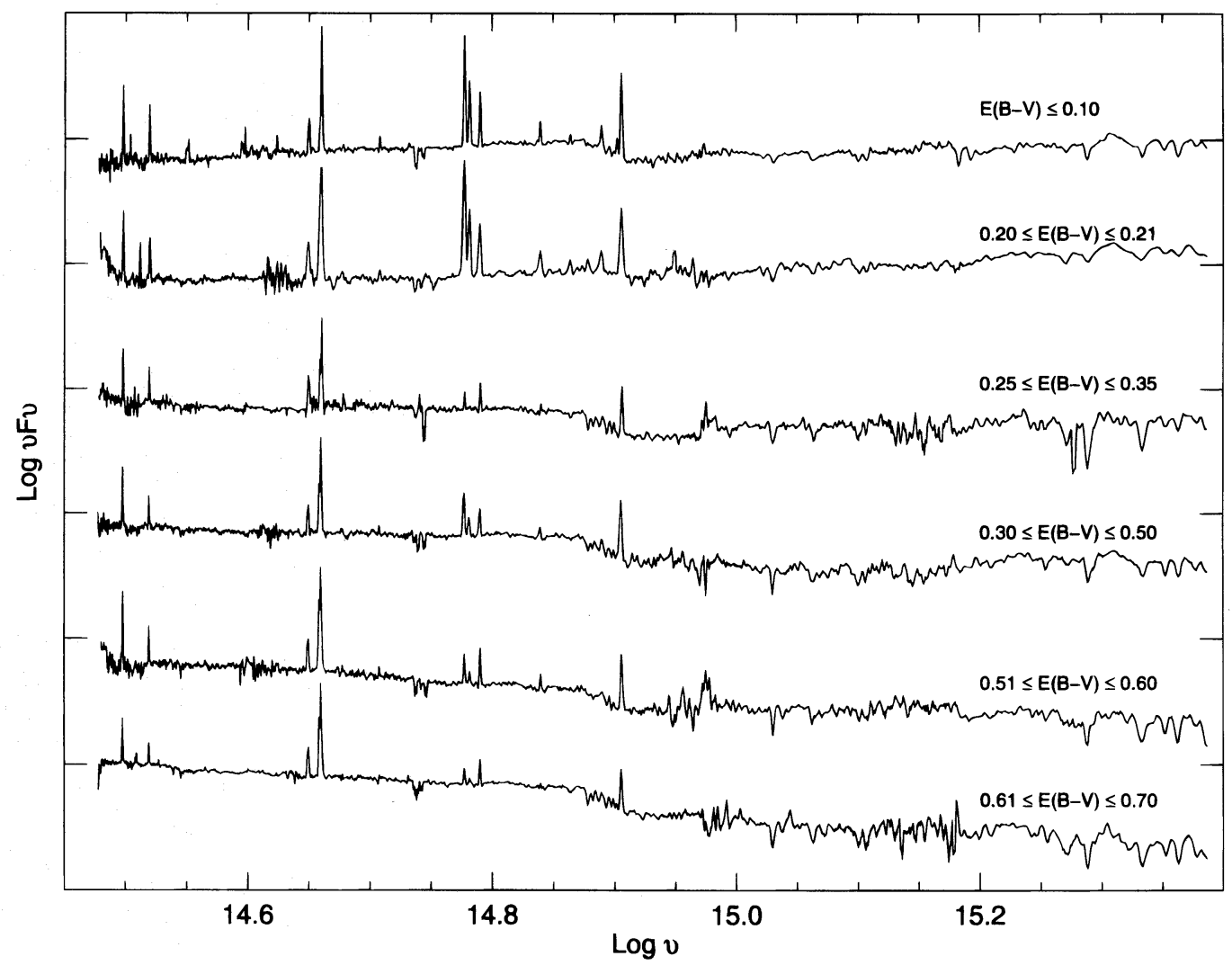

FIG. 13.-Templates of starburst galaxies plotted in the same units as Figs. 10 and 11 

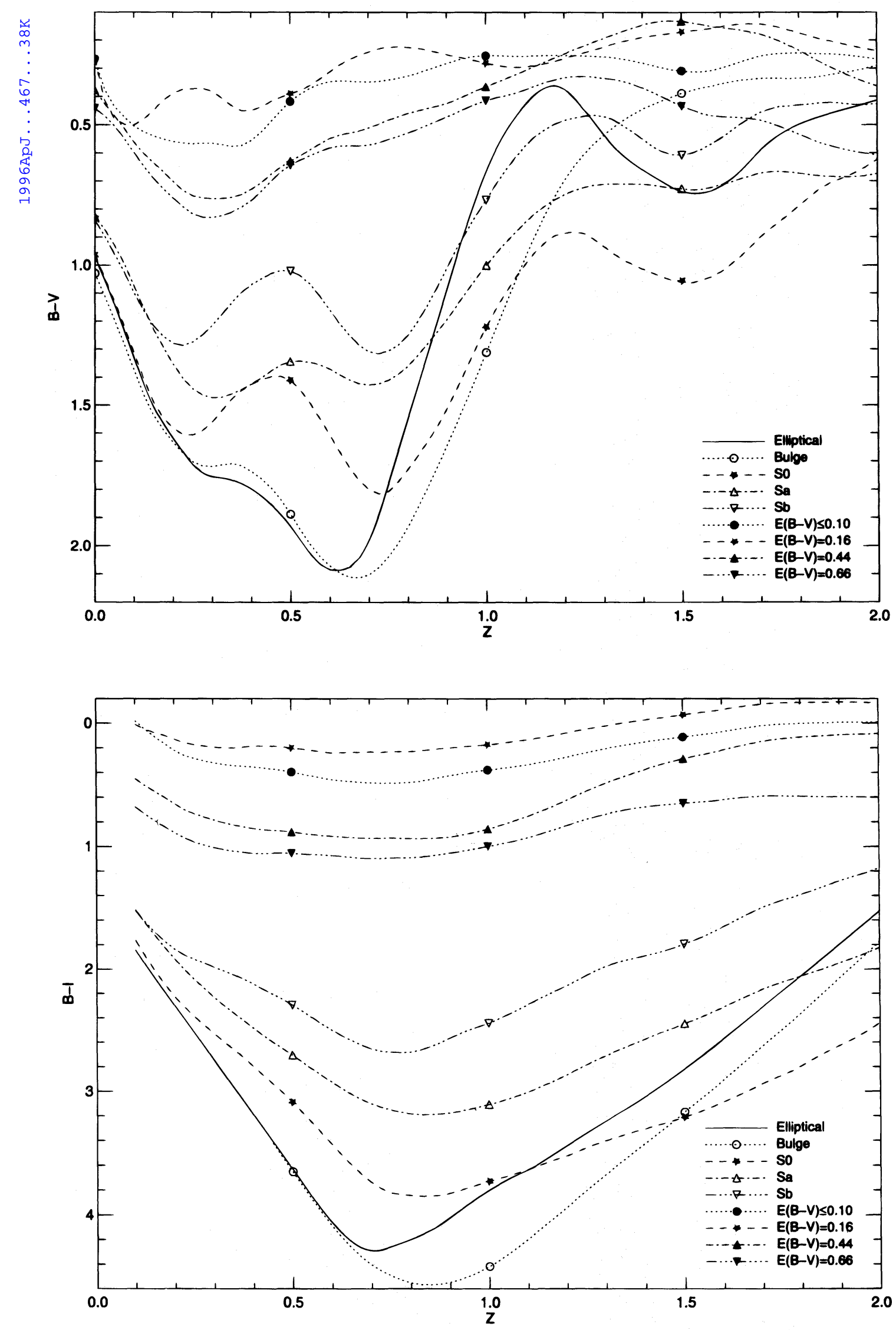

FIG. 14. - Color vs. redshift and color vs. color for the template spectra using the bandpasses shown in Figs. 10 and 11 

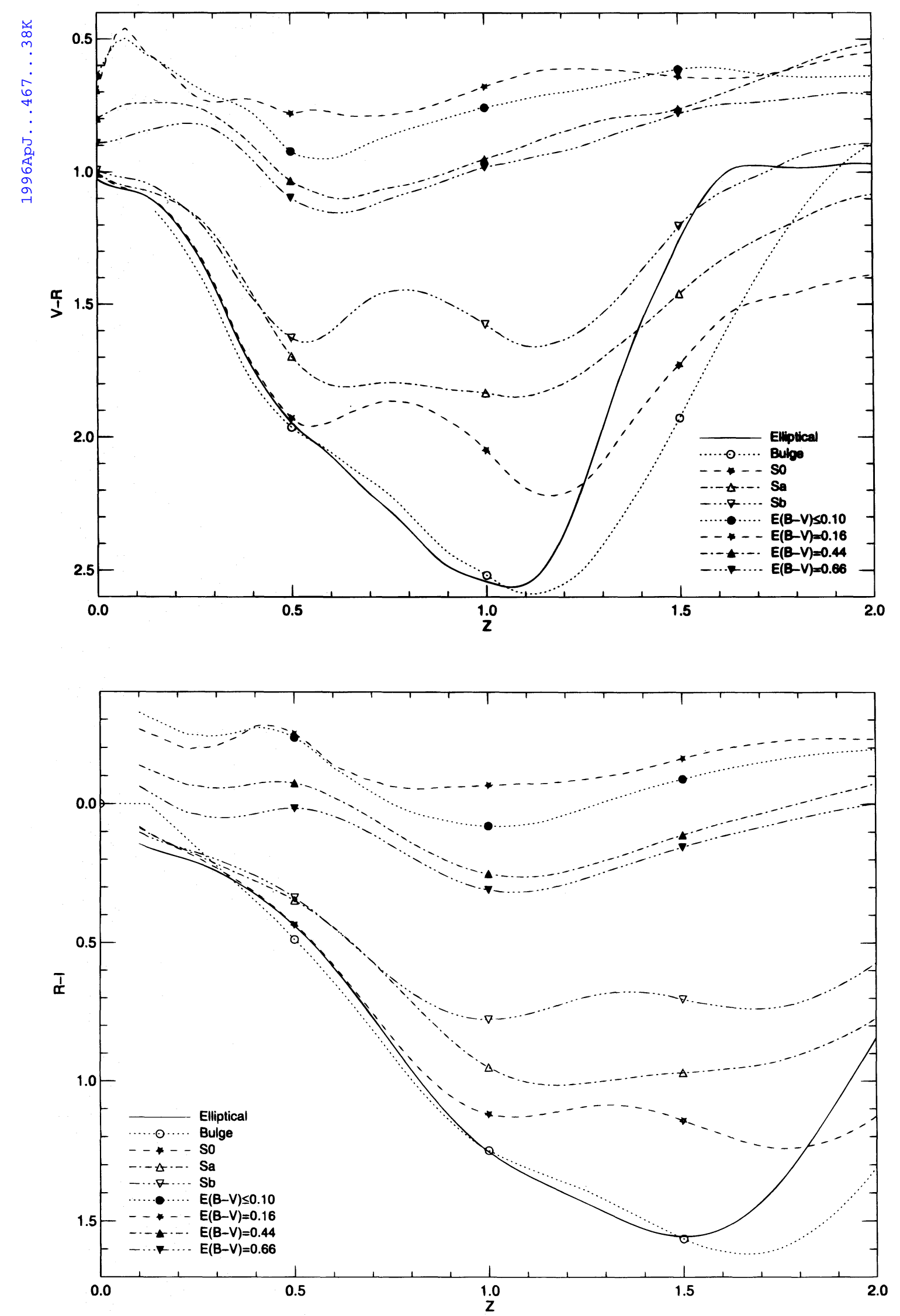

Fig. 14-Continued 

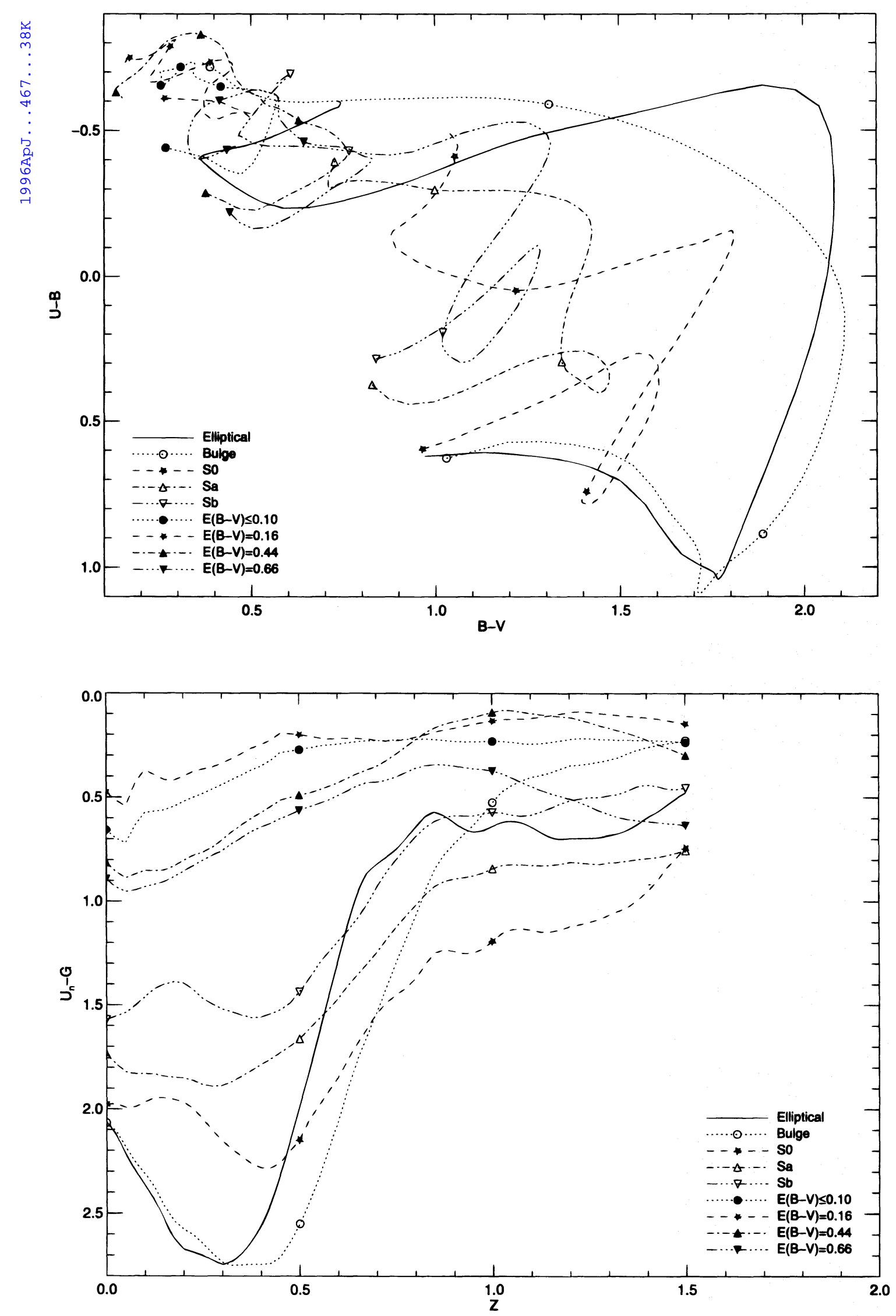

FIG. $14-$ Continued 

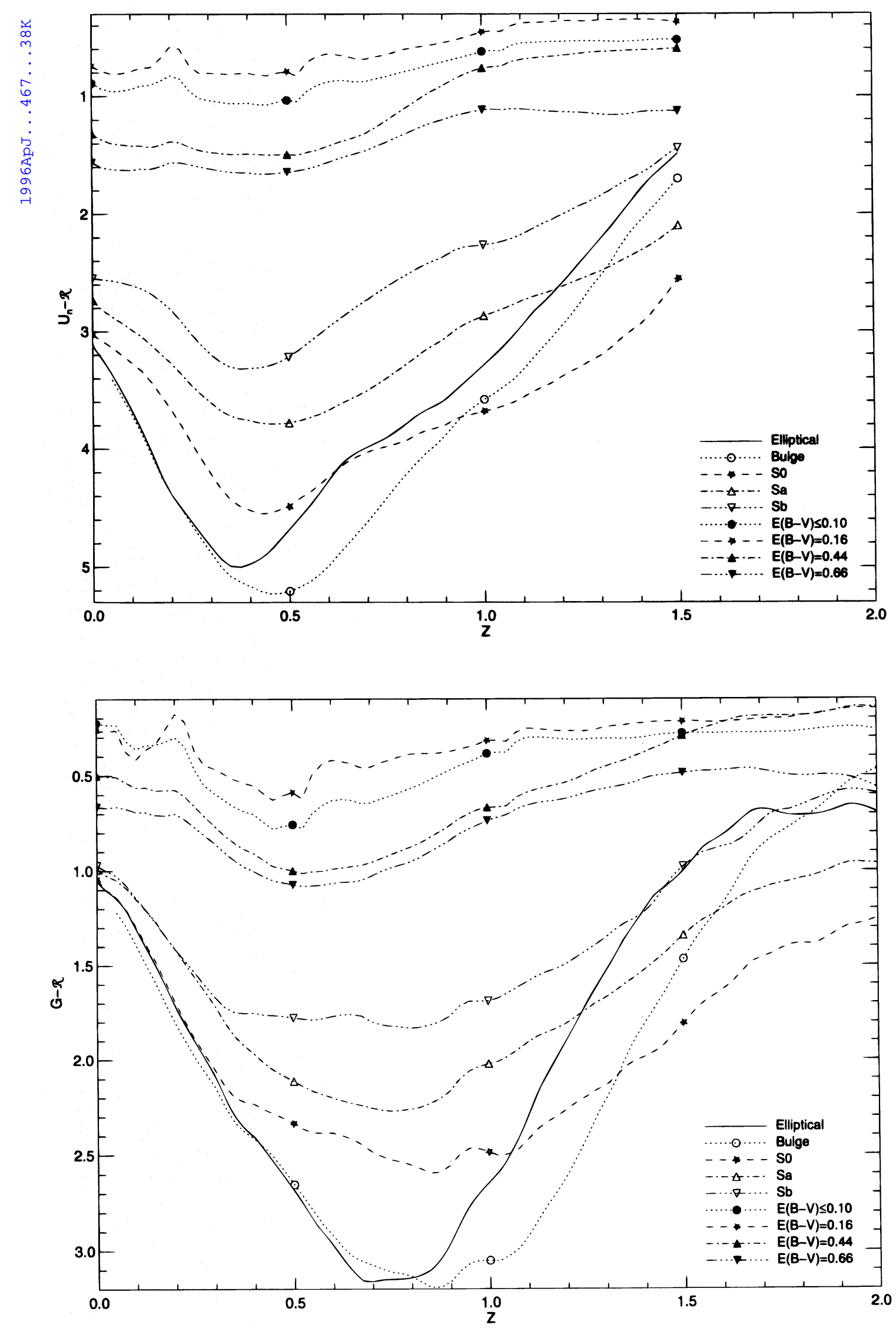

FIG. 14-Continued 

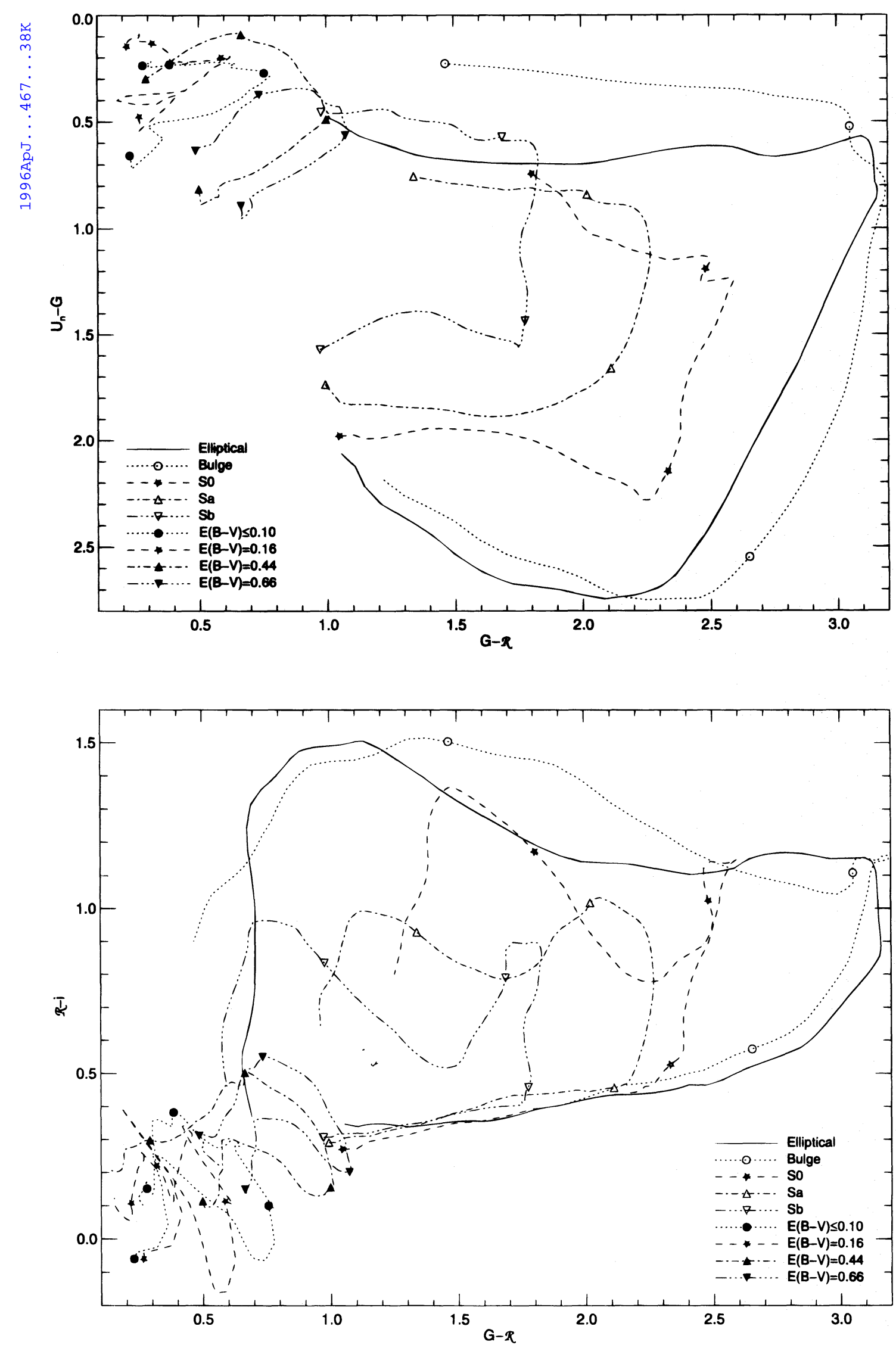

Fig. $14-$ Continued 
TABLE A1 
KINNEY ET AL.

TABLE A1-Continued

\begin{tabular}{|c|c|c|c|c|c|c|c|c|c|c|c|c|}
\hline & Ellip & Bulge & So & $\mathrm{Sa}$ & Sb & $\mathrm{Sc}$ & SB1 & SB2 & SB3 & SB4 & SB5 & SB6 \\
\hline \multirow[t]{2}{*}{$8300-8500$} & 37.009 & & 33.167 & 23.091 & 25.231 & & 5.331 & 5.404 & 8.336 & 8.514 & 11.340 & 10.218 \\
\hline & 0.726 & & 0.781 & 0.378 & 0.533 & & 0.896 & 0.320 & 0.537 & 0.398 & 0.555 & 0.250 \\
\hline \multirow[t]{2}{*}{$8500-8700$} & 36.779 & & 32.644 & 22.779 & 24.784 & & 4.925 & 5.264 & 8.223 & 8.358 & 11.042 & 10.146 \\
\hline & 1.852 & & 1.980 & 1.183 & 1.690 & & 0.304 & 0.352 & 0.481 & 0.511 & 0.963 & 0.576 \\
\hline \multirow[t]{2}{*}{$8700-8900$} & 39.623 & & 35.158 & 24.340 & 26.399 & & 4.913 & 5.381 & 8.748 & 8.639 & 11.319 & 10.758 \\
\hline & 0.527 & & 0.831 & 0.454 & 0.914 & & 0.421 & 0.489 & 0.742 & 0.460 & 0.799 & 0.247 \\
\hline \multirow[t]{2}{*}{$8900-9100$} & 40.073 & & 35.533 & 24.592 & 26.279 & & 5.476 & 6.032 & 9.118 & 8.987 & 11.803 & 11.343 \\
\hline & 0.708 & & 0.950 & 0.543 & 0.722 & & 2.104 & 2.226 & 1.980 & 1.693 & 2.823 & 1.401 \\
\hline \multirow[t]{2}{*}{$9100-9300$} & 39.241 & & 34.490 & 23.501 & 25.652 & & 4.357 & 5.464 & 8.104 & 8.177 & 9.900 & 10.767 \\
\hline & 1.258 & & 1.071 & 0.934 & 1.468 & & 0.572 & 2.139 & 0.866 & 0.677 & 1.150 & 0.888 \\
\hline \multirow[t]{2}{*}{$9300-9500$} & 37.250 & & 32.952 & 22.832 & 24.800 & & 4.781 & 4.929 & 7.745 & 7.748 & 9.851 & 10.451 \\
\hline & 0.958 & & 1.549 & 0.741 & 2.663 & & 1.172 & 0.769 & 1.478 & 0.693 & 1.215 & 0.783 \\
\hline \multirow[t]{2}{*}{$9500-9700$} & 38.234 & & 33.678 & 23.230 & 25.012 & & 5.418 & 6.731 & 9.349 & 9.449 & 11.961 & 11.529 \\
\hline & 0.539 & & 0.830 & 0.425 & 0.880 & & 3.600 & 4.785 & 3.726 & 4.295 & 7.398 & 3.408 \\
\hline \multirow[t]{2}{*}{$9700-9900$} & 38.069 & & 33.669 & 23.206 & 24.779 & & 4.022 & 6.879 & 8.828 & 8.155 & 11.737 & 10.291 \\
\hline & 0.681 & & 0.762 & 0.401 & 1.977 & & 0.754 & 1.498 & 1.111 & 1.036 & 1.973 & 0.588 \\
\hline \multirow[t]{2}{*}{$9900-10000$} & 36.476 & & 33.537 & 22.898 & 24.257 & & 3.975 & 8.041 & 9.219 & 7.557 & 13.070 & 9.036 \\
\hline & 3.542 & & 1.025 & 0.792 & 3.243 & & 0.592 & 1.320 & 1.126 & 1.214 & 0.711 & 1.758 \\
\hline
\end{tabular}

NotE.-See notes to Table 3 for explanation of SB1-SB6.

\section{REFERENCES}

Antonucci, R. R. J. 1993, ARA\&A, 31, 473

Bregman, J. N., Hogg, D. E., \& Roberts, M. S. 1995, ApJ, 441, 561

Broadhurst, T. J. 1989, Ph.D. thesis, Univ, Durham

Broadhurst, T. J., Ellis, R. S., \& Glazebrook, K. 1992, Nature, 335, 55

Broadhurst, T. J., Ellis, R. S., \& Shanks, T. 1988, MNRAS, 235, 827

Bruzual A, G., \& Charlot, S. 1993, ApJ, 405, 538

Burstein, D., Bertola, F., Buson, L. M., Faber, S. M., \& Lauer, T. R. 1988, ApJ, 328, 440

Burstein, D., \& Heiles, C. 1984, ApJS, 54, 33

Calzetti, D., Bohlin, R. C., Kinney, A. L., Storchi-Bergmann, T., \& Heckman, T. M. 1995, ApJ, 443, 136

Calzetti, D., Kinney, A. L., \& Storchi-Bergmann, T. 1994, ApJ, 429, 582

Charlot, S., Worthey, G., \& Bressan, A. 1996, ApJ, 457, 625

Coleman, G. D., Wu, C.-C., \& Weedman, D. W. 1980, ApJS, 43, 393

Condon, J. J., Condon, M. A., Gisler, G., \& Puschell, J. J. 1982, ApJ, 252, 102

Connolly, A. J., Szalay, A. S., Bershady, M. A., Kinney, A. L., \& Calzetti, D. 1995, AJ, 110, 1071

Fabbiano, G. 1989, ARA\&A, 27, 87

Fabbiano, G., Kim, D.-W., \& Trinchieri, G. 1992, ApJS, 80, 531

Giavalisco, M., Macchetto, F. D., \& Sparks, W. B. 1994, A\&A, 288, 103

Gunn, J. E., \& Knapp, G. R. 1992, PASP, 43, 267

Israel, F. P., \& van der Hulst, J. M. 1983, AJ, 88, 1736

Kennicutt, R. C. 1992, ApJS 79, 255

King, C. R., \& Ellis, R. S. 1985, ApJ, 288, 456

Kinney, A. L., Bohlin, R. C., Calzetti, D., Panagia, N., \& Wyse, R. F. G. 1993, ApJS, 86, 5
Koo, D. C., \& Kron, R. G. 1992, ARA\&A, 30, 613

Koratkar, A. P., Bohlin, R. C., Calzetti, D., \& Kinney, A. L. 1996, in preparation

Lawrence, A., \& Elvis, M. 1982, ApJ, 256, 410

Leitherer, C., \& Heckman, T. M. 1995, ApJS, 96, 38

McQuade, K., Calzetti, D., \& Kinney, A. L. 1995, ApJS, 97, 331

Pedlar, A., Booler, R. V., Specer, R. E., \& Stewart, O. J. 1983, MNRAS, 202, 647

Pence, W. 1976, ApJ, 203, 39

Pier, E., \& Krolik, J. 1992, ApJ, 401, 99

Roche, P. F., Aitken, D. K., Smith, C. H., \& Ward, M. J. 1991, MNRAS, 248,606

Sadler, E. M. 1984, AJ, 89, 53

Sanders, D. B., Phinney, E. S., Neugebauer, G., Soifer, B. T., \& Mathews, K. 1989, ApJ, 347, 29

Schechter, P. 1976, ApJ, 203, 297

Seaton, M. J. 1979, MNRAS, 187, 73P

Steidel, C. C., \& Hamilton, D. 1992, AJ, 104, 941 1993, 105, 2017

Storchi-Bergmann, T., Kinney, A. L., \& Challis, P. 1995, ApJS, 98, 103

Subrahmanya, C. R., \& Harnett, J. I. 1987, MNRAS, 225, 297

Ulvestad, J. S., \& Wilson, A. S. 1984, ApJ, 285, 439

Weedman, D. W., Feldman, F. R., Balzano, V. A., Ramsey, L. W., Sramek, R. A., \& Wu, C.-C. 1981, ApJ, 248, 105

Wirtz, V. C. 1918, Astron. Nachr., 206, 109 